\title{
Reduced order models for geometrically nonlinear structures: assessment of implicit condensation in comparison with invariant manifold approach
}

\author{
Yichang Shen ${ }^{\mathrm{a}}$, Natacha Béreux ${ }^{\mathrm{a}}$, Attilio Frangi ${ }^{\mathrm{b}}$, Cyril Touzéa ${ }^{\mathrm{a}, *}$ \\ aIMSIA, CNRS, EDF, CEA, ENSTA Paris, Institut Polytechnique de Paris, 828 Boulevard des Maréchaux, 91762 Palaiseau \\ Cedex, France \\ ${ }^{b}$ Departement of Civil and Environmental Engineering, Politecnico di Milano, Milano, Italy
}

\begin{abstract}
A comparison between two methods to derive reduced-order models (ROM) for geometrically nonlinear structures is proposed. The implicit condensation and expansion (ICE) method relies on a series of applied static loadings. From this set, a stress manifold is constructed for building the ROM. On the other hand, nonlinear normal modes rely on invariant manifold theory in order to keep the key property of invariance for the reduced subspaces. When the model coefficients are fully known, the ICE method reduces to a static condensation. However, in the framework of finite element discretisation, getting all these coefficients is generally too computationally expensive. The stress manifold is shown to tend to the invariant manifold only when a slow/fast decomposition between master and slave coordinates can be assumed. Another key problem in using the ICE method is related to the fitting procedure when a large number of modes need to be taken into account. A simplified procedure, relying on normal form theory and identification of only resonant monomial terms in the nonlinear stiffness, is proposed and contrasted with the current method. All the findings are illustrated on beams and plates examples.
\end{abstract}

Keywords: model order reduction, nonlinear normal mode, geometric nonlinearity, invariant manifold, implicit condensation and expansion

\section{Introduction}

When vibrating with large amplitudes, thin structures such as beams, plates and shells experience geometric nonlinearity due to the nonlinear relationship between strains and displacements $[1,2,3]$. In turn, this may give rise to a number of nonlinear phenomena that have no counterparts in linear vibrations: jump phenomena [4], sub and superharmonic resonances [5], quasiperiodic regimes [6,7], strong nonlinear couplings due to internal resonances $[8,9,10]$, chaotic vibrations $[11,12]$, and appearance of a continuous spectrum highlighting the presence of wave turbulence $[13,14,15]$. Most of these nonlinear effects create strong couplings and energy exchange in the frequency domain, so that the number of modes required to simulate these dynamical solutions are far larger than what could be expected from linear theory. As a consequence, the derivation of efficient reduced-order models (ROMs) has always been a topic of interest.

In the past decades, numerous methods have been proposed. A number of them rely on a linear change of coordinates: Ritz vectors [16], Proper orthogonal decomposition (POD) [17, 18, 19], as well as the proper generalized decomposition (PGD) [20, 21]. A large body of work has been devoted to geometrically nonlinear models expressed with simplifying assumptions such as von Kármán models for beams and plates [22] or Donnell variants for shells [7, 23]. In this case the equations of motions are known as partial differential equations (PDE), and methods from the applied mathematics community can be directly used. This gives rise in particular to all the developments on nonlinear normal modes

${ }^{*}$ Corresponding author

Email addresses: yichang.shen@ensta-paris.fr(Yichang Shen), natacha.bereux@edf.fr (Natacha Béreux), attilio.frangi@polimi.it (Attilio Frangi), cyril.touze@ensta-paris.fr (Cyril Touzé) 
(NNMs), defined as invariant manifolds in phase space [24, 25, 26]. In the same line, the normal form theory has also been used in order to derive efficient ROMs with normal coordinates linked to the invariant manifolds $[27,28,29]$. More recently, spectral submanifolds have been defined in order to get a more generic and mathematically rigorous definitions of the reduction subspaces that could be used independently for conservative or dissipative mechanical systems [30, 31].

When the structure of interest is discretized with the finite element (FE) approach, as is routinely used nowadays in engineering, the analyst cannot rely on a PDE to implement its favourite reduced basis. Consequently, a large body of literature has been developed in order to cope with the specificities of finite-element based formulations. One of the main problem in this case is to derive first a model that can be used for processing a reduction method. In this respect, numerous studies have been oriented towards non-intrusive methods, capable of computing the nonlinear coefficients of the geometrically nonlinear restoring force. By non-intrusive, it is meant that the methods can be used with any commercial FE software without the need of coding a computation at the level of the element [32], contrary to the so-called direct methods [33,34]. Among these, two main families of methods have been proposed. The first one relies on applying a series of static prescribed displacements, and has been called STEP for Stiffness Evaluation Procedure. From the static displacements, a simple algebra shows that one can directly retrieve the modal nonlinear coupling coefficients [35, 36, 37]. The second one relies on applying a series of static selected forces, and has been called the implicit condensation and expansion (ICE) method [38, 39, 40]. From the loadings and resulting large-amplitude displacements, a so-called stress manifold can be constructed in order to reduce the dynamics [41]. A main advantage of these two procedures (STEP and ICE) thus relies in their versatility and ease-of-use due this non-intrusive characteristics.

Despite the number of studies concerned with reduced-order models, very few of them have been devoted to explaining the connections between the methods developed independently by two different communities and briefly sketched in the previous paragraphs. Kuether and Allen addressed the computation of the backbone curves (or amplitude-frequency relationship, also defined as NNM in a conservative framework) for ROMs built with the ICE method [42], but without clearly evidencing the relationship between the two methods. In the same lines, the backbone curves have been computed with ROMs built from modal derivatives in [43], but the exact connection between invariant manifold and quadratic manifold built from modal derivatives has been reported in [44]. The aim of this contribution is thus to analyse the relationship between the stress manifolds produced with the ICE method, and the invariant manifolds defining NNMs. Since the invariance property is key in order to define an efficient ROM (otherwise the trajectories produced by the ROM will not exist for the full system), understanding in the phase space of the system, how these two reduced subspaces compares, is key. This will be achieved thanks to analytical calculations and examples on a simple two degrees-of-freedom system, and will assess the quality and limitations of the ICE method in producing efficient ROMs for geometrically nonlinear structures.

The paper is organized as follows. Section 2 recalls the theoretical settings and explains the ICE method and the invariant manifold approach. In particular, it is shown that in the specific case where all the coefficients of the model are fully known, then the ICE method simplifies to the standard static condensation of current use to simplify the high-frequency components of a vibrating structure. From a theoretical point of view, contrasting the two methods is then equivalent to comparing static condensation with NNM-based reduction. It is then shown in a general framework that if one assumes a slow/fast decomposition between the master and slave coordinates, then the static condensation approach reduces to the invariant manifold approach, at the leading order. Section 3 confirms these general findings by simplifying to a two degrees-of-freedom (dofs) system, for which explicit analytical expressions can be derived thanks to asymptotic expansions, leading to an accurate term-by-term comparison. Section 4 extends the results to the case of continuous structures. Beam examples with plate and three-dimensional elements are tested, as well as a plate with simply supported boundary conditions. In this section, a thorough discussion on the construction of multi-mode ROMs with the ICE method is given, and a proposition in order to reduce the burden of the fitting procedure, is developed. Using normal form theory and the recognition that only resonant monomial terms have a strong influence on the dynamics, 
it is proposed to fit only these resonant monomials in the nonlinear restoring force.

\section{Theoretical settings}

\subsection{Framework}

Thin structures experiencing large displacements are considered, so that geometrical nonlinearities are excited. The usual framework assumes also small strains and a linear behaviour law, so that the relationship between the second Piola Kirchhoff stress tensor $\mathbf{S}$ and the the Green-Lagrange strain tensor G reads, following the Saint-Venant Kirchhoff law [45, 1] :

$$
\mathbf{S}=\mathbf{A}: \mathbf{G}, \quad \text { with } \quad \mathbf{G}=\frac{1}{2}\left(\nabla \mathbf{u}+\nabla^{t} \mathbf{u}+\nabla^{t} \mathbf{u} \cdot \nabla \mathbf{u}\right),
$$

where $\mathbf{A}$ is the tensor of constant stiffness terms describing the material law, and $\mathbf{u}$ is the three-dimensional displacement. The weak form is then written in the reference configuration and reads:

$$
\int_{\Omega_{0}} \rho \ddot{\mathbf{u}} \cdot \mathbf{w} \mathrm{d} \Omega+\int_{\Omega_{0}} \mathbf{P}: \nabla^{t} \mathbf{w} \mathrm{d} \Omega=\int_{\Omega_{0}} \rho \mathbf{F} \cdot \mathbf{w} \mathrm{d} \Omega+\int_{\mathcal{S}_{0}} \mathbf{f} \cdot \mathbf{w} \mathrm{d} \mathcal{S}, \quad \forall \mathbf{w} \in C^{0},
$$

where $\mathbf{w}$ is a continuous test function, $\mathbf{P}$ is the first Piola-Kirchhoff stress tensor, $\mathbf{P}=(1+\nabla \mathbf{u}) \cdot \mathbf{S}$, and $\rho \mathbf{F}$ and $\mathbf{f}$ are respectively volumic and surface external forces exerted on the body occupying the domain $\Omega_{0}$ with boundary surface $\mathcal{S}_{0}$.

Most of the developments in this article are concerned with a space discretization relying on the finite element $(\mathrm{FE})$ procedure. In that context, the equivalent semi-discrete, finite dimensional expression of Eq. (2), generally reads:

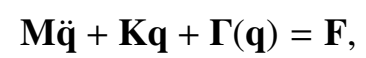

with $\mathbf{q}$ the vector of generalized displacements (displacements at the nodes) with dimension $N, \mathbf{M}$ the mass matrix, $\mathbf{K}$ the tangent stiffness matrix, $\boldsymbol{\Gamma}(\mathbf{q})$ representing the nonlinear restoring force and $\mathbf{F}$ the external forces. It can be shown in particular that in the context of geometrically nonlinear structures, the nonlinear part of the stiffness $\boldsymbol{\Gamma}(\mathbf{q})$ is polynomial and contains only quadratic and cubic terms $[32,34,46]$.

The linear modal basis is defined by the vectors $\phi_{i}$ such that

$$
\mathbf{K} \phi_{i}=\omega_{i}^{2} \mathbf{M} \phi_{i} .
$$

In the modal basis, the equations of motion can be rewritten, using the linear change of coordinates $\mathbf{q}=\mathbf{P}_{\phi} \mathbf{X}$, with $\mathbf{P}_{\phi}$ the matrix of eigenvectors and $\mathbf{X}$ the modal coordinates :

$$
\ddot{X}_{p}+\omega_{p}^{2} X_{p}+\sum_{i=1}^{N} \sum_{j \geq i}^{N} g_{i j}^{p} X_{i} X_{j}+\sum_{i=1}^{N} \sum_{j \geq i}^{N} \sum_{k \geq j}^{N} h_{i j k}^{p} X_{i} X_{j} X_{k}=\tilde{F}_{p},
$$

with $\tilde{\mathbf{F}}=\mathbf{P}_{\phi}^{t} \mathbf{F}$ the vector of modal forcings, $g_{i j}^{p}$ and $h_{i j k}^{p}$ the quadratic and cubic nonlinear coupling coefficients. This formulation is exact in the case of a structure discretized with FE $[32,34,46]$ assuming geometric nonlinearity, as well as for simplified models based on von Kármán assumptions for beams, plates and shells [46, 22]. For the sake of simplicity, the modal equations are also rewritten as:

$$
\ddot{X}_{p}+\omega_{p}^{2} X_{p}+f_{p}\left(X_{1}, X_{2}, \ldots, X_{N}\right)=\tilde{F}_{p} .
$$

The main problem when using a FE procedure is that the nonlinear coupling coefficients are a priori not available, and require an additional direct or indirect method to compute them [32, 34, 33]. Also the number of modes can be prohibitively large to derive reduced-order models, as underlined in e.g. $[46,47]$. Consequently ad-hoc methods are given in the literature in order to overcome these problems and directly propose reduced-order models with a limited number of selected coordinates and number of coupling coefficients to compute. The aim of this study is to compare the implicit condensation method with nonlinear normal modes. We begin with a description of the ICE method. 


\subsection{ICE method and stress manifold}

The Implicit Condensation and Expansion (ICE) method has been first introduced by McEwan, Gordon and Hollkamp [38, 39, 48, 40], and recently used by Kuether et al. [42], and Frangi and Gobat [41]. As compared to the STEP method, where a series of static prescribed displacements are used in order to get the values of the modal coupling coefficients $g_{i j}^{p}$ and $h_{i j k}^{p}$ given in Eq. (5), the ICE method relies on a series of applied static forcings. Whereas the STEP method gives a direct access to the modal coupling coefficients, the values obtained with the ICE method are different and depend on the level of applied force and are thus load dependent. This is directly related to the fact that the coordinates used to build ROMs from the ICE method are not the modal coordinates. Instead, a stress manifold is built from the series of applied loads, and new coordinates are used to describe the dynamics onto this manifold. In other words, ICE method allows to directly pass from the physical space (nodes of the FE structure) to the curved stress manifold and the reduced variables describe the dynamics onto this stress manifold; without resorting to an intermediate step where modal coordinates are needed. Since the stress manifold is curved and amplitude-dependent, it is fully logical to obtain coefficients that are load-dependent. On the other hand, if one refers to the STEP applied to eigenmodes, then the computed coupling coefficients should not depend on amplitude since related to planar linear eigensubspaces. This explains why the coefficients from STEP do not depend upon amplitude in the large range of amplitude where the nonlinearity is correctly excited, see e.g. [46]. Using applied forces instead of prescribed displacements allows one to get a better track of how the nonlinear couplings generated by the nonlinear internal force transfer energies between oscillators. But the drawback of the method relies on the fact that a fitting procedure is needed after application of the forces in order to get the nonlinear restoring force on the stress manifold.

The procedure is as follows. First one imposes body forces $\mathbf{F}$ that are proportional to the inertia of the linear modes, $\mathbf{F}=\beta_{i} \phi_{i}(\mathbf{x})$ in Eq. (3), for a selected number $i$ of modes, $i=1 \ldots m$, where $m$ is the number of modes selected in the final ROM. A static problem is then solved and the obtained displacement field is projected onto the eigenmodes in order to get the modal displacements $X_{p}$ corresponding to the imposed force. A mapping is thus constructed with entries $\beta_{i}$ and outputs $X_{p}$. Assuming that the stress manifold can be inverted, one obtains $X_{p}\left(\beta_{i}\right)$, from which the ROM can be built. For this last step, a fitting procedure is needed so as to derive functional forms from the computed clouds of points.

As noted by different authors [40, 42], the method allows to make an implicit condensation of the non modeled degrees of freedom. The procedure is thus particularly appealing when working with thin structures, in order to implicitly take into account the axial-bending nonlinear coupling that gives rise to geometric nonlinearities. One may also notice that, in the specific cases of small models where the equations of motion are fully known (i.e. if one is able to get the full model equations as in (5) for all the degrees of freedom, which is generally out of reach for complex structures meshed with FE), the implicit condensation becomes explicit, so that the method is equivalent to the usual static condensation.

In the context of drawing out a full comparison of ICE method with NNMs, we will first use full models where all coefficients are known, so that ICE method reduces to static condensation. Let us first separate the degrees of freedom of the modal displacement $\mathbf{X}$ between the first $m$ master coordinates $X_{1}, \ldots, X_{m}$, and the remaining slave coordinates $X_{m+1}, \ldots, X_{N}$. Let us also assume that for the slave coordinate $s \in[m+1, N]$, inertia $\ddot{X}_{s}$ can be neglected. This hypothesis is generally justified by the fact that the slave coordinates correspond to high-frequency modes with fast oscillations. The method then assumes that a functional relationship $c_{s}$ exists between the master coordinates and the slave coordinates:

$$
\forall s \in[m+1, N], \quad X_{s}=c_{s}\left(X_{1}, X_{2}, \ldots, X_{m}\right) .
$$

These relationships define the stress manifold in phase space, and only depend on the displacements. The functions $c_{s}$ are determined from:

$$
\forall s \in[m+1, N], \quad \omega_{s}^{2} c_{s}\left(X_{1}, \ldots, X_{m}\right)+f_{s}\left(X_{1}, \ldots, X_{m}, c_{m+1}\left(X_{1}, \ldots, X_{m}\right), \ldots, c_{N}\left(X_{1}, \ldots, X_{m}\right)\right)=0 .
$$

then, the reduced-order model for the master coordinates reads:

$$
\forall t \in[1, m], \quad \ddot{X}_{t}+\omega_{t}^{2} X_{t}+f_{t}\left(X_{1}, \ldots, X_{m}, c_{m+1}\left(X_{1}, \ldots, X_{m}\right), \ldots, c_{N}\left(X_{1}, \ldots, X_{m}\right)\right)=0 .
$$




\subsection{Invariant manifolds}

In this article, we use the definition of NNMs as proposed by Shaw and Pierre [24, 49, 50, 25]. The method relies on the center manifold theorem [51, 52], and allows a clear conceptual definition of an NNM as an invariant manifold in phase space, tangent to the linear eigenspaces at the origin. One of the main advantages of the method is to provide a clear continuation of linear eigenspaces to nonlinear regimes, as well as giving efficient reduced-order subspaces for building ROMs since the key property of invariance is conserved. Application of the method to full FE models has been however rarely discussed in the literature, see e.g. [53] for an example. The main reason lies in the fact that application of the method as it was presented in $[24,49,26]$ needs as input the nonlinear coupling coefficients, obtained for example from a STEP. However, recent developments overcome this limitation, see e.g. [54] for a direct approach using spectral submanifolds (with general third-order formula equivalent to the ones given with the invariant manifold method proposed by Shaw and Pierre), and $[55,56]$ for a direct method based on normal form.

The starting point is to put Eqs. (6) in first-order form (dynamical system), for the $r$ th mode:

$$
\begin{aligned}
& \dot{X}_{r}=Y_{r}, \\
& \dot{Y}_{r}=-\omega_{r}^{2} X_{r}-f_{r}\left(X_{1}, \ldots, X_{N}\right) .
\end{aligned}
$$

The invariant manifold is defined by functional relationships $a_{s}$ and $b_{s}$ relating the slave coordinates to the master ones by, $\forall s \in[m+1, N]$ :

$$
\begin{aligned}
X_{s} & =a_{s}\left(X_{1}, Y_{1}, \ldots, X_{m}, Y_{m}\right), \\
Y_{s} & =b_{s}\left(X_{1}, Y_{1}, \ldots, X_{m}, Y_{m}\right) .
\end{aligned}
$$

Following the guidelines of the center manifold theorem, one arrives at the two following equations, describing the geometry of the $2(N-m)$ dimensional invariant manifold in phase space :

$$
\begin{aligned}
& \sum_{t=1}^{m}\left(\frac{\partial a_{s}}{\partial X_{t}} Y_{t}+\frac{\partial a_{s}}{\partial Y_{t}}\left[-\omega_{t}^{2} X_{t}-f_{t}\right]\right)=b_{s} \\
& \sum_{t=1}^{m}\left(\frac{\partial b_{s}}{\partial X_{t}} Y_{t}+\frac{\partial b_{s}}{\partial Y_{t}}\left[-\omega_{t}^{2} X_{t}-f_{t}\right]\right)=-\omega_{s}^{2} a_{s}-f_{s}
\end{aligned}
$$

The solutions of these partial differential equations are generally difficult to obtain. No analytical solutions exist and the early developments proposed asymptotic expansions in order to compute the first nonlinear dependences of invariant manifolds upon amplitudes [24, 49, 50, 25]. Numerical methods have also been formulated to get effective ROMs based on invariant manifolds up to very large amplitudes $[26,57,58,59]$. Whatever the method, once the unknown functions $a_{s}$ and $b_{s}$ have been obtained, the dynamics of the ROM reads:

$$
\forall t \in[1, m], \quad \ddot{X}_{t}+\omega_{t}^{2} X_{t}+f_{t}\left(X_{1}, \ldots, X_{m}, a_{m+1}\left(X_{1}, Y_{1}, \ldots, X_{m}, Y_{m}\right), \ldots, a_{N}\left(X_{1}, Y_{1}, \ldots, X_{m}, Y_{m}\right)\right)=0 .
$$

One can note that the static condensation and invariant manifold approaches share similarities in the way reduced-order models are derived. However, this general presentation shows that the invariant manifold approach appears to be more general. Indeed, whereas static condensation works out only on displacements, the invariant manifold approach includes also the velocities as independent variables, so that two unknown functions are to be found, each of which depending on two master coordinates. Furthermore, no assumption on neglecting any inertia is introduced when computing the NNMs. This suggests that the two methods should have similarities only in the case where a clear slow/fast decomposition holds between slave and master coordinates, and that the stress manifold would not be able to take into account internal resonance relationship, a key feature in nonlinear vibrations. Finally, the NNM approach allows conserving the key property of invariance for the reduced subspaces, an attribute that is not embedded in the stress manifold. 


\subsection{Slow/fast decomposition}

Let us assume that a slow/fast decomposition of the system is at hand, which means that the slave coordinates $X_{s}$, for $s \in[m+1, N]$, have a radian eigenfrequency $\omega_{s}$ which is much larger than those of the master coordinates $X_{t}$, for $t \in[1, m]: \omega_{s} \gg \omega_{t}$. In order to take this assumption into account in the equations of motion, one can introduce a small parameter $\varepsilon$ and scale the linear and nonlinear restoring forces of the slave variables by $1 / \varepsilon$ in order to express the fact that the slave coordinates are much more stiff and thus corresponds to fast oscillations. Therefore the dynamics of the system (6) without external forcing, can be rewritten as:

$$
\begin{array}{cl}
\forall t \in[1, m], & \ddot{X}_{t}+\omega_{t}^{2} X_{t}+f_{t}\left(X_{1}, X_{2}, \ldots, X_{N}\right)=0, \\
\forall s \in[m+1, N], & \ddot{X}_{s}+\frac{1}{\varepsilon} \omega_{s}^{2} X_{s}+\frac{1}{\varepsilon} f_{s}\left(X_{1}, X_{2}, \ldots, X_{N}\right)=0 .
\end{array}
$$

With this formulation, Eq. (14b) justifies the assumption of neglecting the inertia of the fast variable so that one arrives easily at Eq. (8) allowing the computation of the stress manifold.

The equations describing the geometry of the invariant manifold, Eqs. (12), rewritten with the slow/fast assumption, read:

$$
\begin{aligned}
& \sum_{t=1}^{m}\left(\frac{\partial a_{s}}{\partial X_{t}} Y_{t}+\frac{\partial a_{s}}{\partial Y_{t}}\left[-\omega_{t}^{2} X_{t}-f_{t}\right]\right)=b_{s}, \\
& \sum_{t=1}^{m}\left(\frac{\partial b_{s}}{\partial X_{t}} Y_{t}+\frac{\partial b_{s}}{\partial Y_{t}}\left[-\omega_{t}^{2} X_{t}-f_{t}\right]\right)=-\frac{1}{\varepsilon} \omega_{s}^{2} a_{s}-\frac{1}{\varepsilon} f_{s} .
\end{aligned}
$$

Eq. (15b) shows that thanks to the slow/fast assumption, $a_{s}$ is, at first order, solution of the following, obtained by neglecting the $\varepsilon$ terms:

$$
\forall s \in[m+1, N], \quad \omega_{s}^{2} a_{s}\left(X_{1}, Y_{1}, \ldots, X_{m}, Y_{m}\right)+f_{s}\left(X_{1}, \ldots, X_{m}, a_{m+1}, \ldots, a_{N}\right)=0 .
$$

This equation is completely equivalent to Eq. (8), showing that with the slow/fast assumption, the function $a_{s}$ shall thus tend to the $c_{s}$ obtained with the static condensation, the only difference being the dependence on the velocities of $a_{s}$, that is not assumed for the $c_{s}$ functions. Based on this observation, one can also assume that the $a_{s}$, as being solutions of the same problem as the $c_{s}$, will not depend on the velocities, so that $\forall t \in[1, m], \partial a_{s} / \partial Y_{t}=0$. Reporting this in Eq. (15a) shows that a simple relationship should hold between $a_{s}$ and $b_{s}$ as:

$$
b_{s}=\sum_{t=1}^{m} \frac{\partial a_{s}}{\partial X_{t}} Y_{t}
$$

All these relationships shows that static condensation and invariant manifolds should propose equivalent results when a slow/fast decomposition of the system can be assumed. However, if the slave modes are not stiff as compared to the master, then the ICE method should be used with care, and a better approach is to use invariant manifolds in order to propose efficient ROMs.

\subsection{Type of nonlinearity}

In this section, we compare how both methods allow predicting the type of nonlinearity, defined as the hardening/softening dependence of the oscillation frequency upon amplitude. Indeed, the ability of a method to correctly predict the backbone curve of a nonlinear oscillator is key, and reduced-order models need to be able to give a correct prediction at least to the first order. For that purpose, let us assume that a single master coordinate, label $p$, is retained, all other coordinates for $s \neq p$ belonging to the slave variables. The reduced-order model will then consist of a single oscillator equation from which one can derive the type of nonlinearity. Also, the leading order term that dictates the hardening/softening behaviour can be found from a perturbative solution where the single nonlinear oscillator equation is truncated to the cubic order [4, 27]. Consequently the general equations describing the stress manifolds, the invariant manifolds, and the dynamics within them, can be truncated up to order three. 
In the case of the static condensation, one has to solve Eq. (7) up to quadratic term only since, when replacing in Eq. (9), the slave coordinates are at least of second order. The first-order term for Eq. (7) is easy to derive and one can write immediately, for all $s \neq p$

$$
X_{s}=c_{s}\left(X_{p}\right) \simeq-\frac{g_{p p}^{s}}{\omega_{s}^{2}} X_{p}^{2}+O\left(X_{p}^{3}\right) .
$$

Consequently the dynamics of the master mode on the stress manifold reads

$$
\ddot{X}_{p}+\omega_{p}^{2} X_{p}+g_{p p}^{p} X_{p}^{2}+\left(h_{p p p}^{p}-\sum_{\substack{s=1 \\ s \neq p}}^{N} \frac{g_{p s}^{p} g_{p p}^{s}}{\omega_{s}^{2}}\right) X_{p}^{3}+O\left(X_{p}^{4}\right)=0
$$

The frequency-amplitude relationship can be derived from this equation by a perturbative approach, see e.g. $[4,27]$. One arrives at the following generic formula

$$
\omega_{\mathrm{NL}}=\omega_{p}\left(1+\Gamma a^{2}\right),
$$

where $\omega_{\mathrm{NL}}$ is the nonlinear frequency, depending on amplitude $a$, and $\Gamma$ is a coefficient dictating the type of nonlinearity (hardening oscillator for $\Gamma>0$, softening for $\Gamma<0$ ). Applying the first-order formula from a perturbation method to Eq. (19), the type of nonlinearity for the static condensation approximation $\Gamma_{\mathrm{SC}}$ reads

$$
\Gamma_{\mathrm{SC}}=\frac{1}{8 \omega_{p}^{2}}\left(3 h_{p p p}^{p}-\frac{10\left(g_{p p}^{p}\right)^{2}}{3 \omega_{p}^{2}}-\sum_{\substack{s=1 \\ s \neq p}}^{N} \frac{3 g_{p s}^{p} g_{p p}^{s}}{\omega_{s}^{2}}\right) .
$$

In the case of the invariant manifold approach, Eqs. (11) can be rewritten with the assumption of a single master NNM, for all $s \neq p$, and up to the second order:

$$
\begin{aligned}
& X_{s}=a_{s}\left(X_{p}, Y_{p}\right)=A_{s, 1}^{p} X_{p}^{2}+A_{s, 2}^{p} X_{p} Y_{p}+A_{s, 3}^{p} Y_{p}^{2}, \\
& Y_{s}=b_{s}\left(X_{p}, Y_{p}\right)=A_{s, 4}^{p} X_{p}^{2}+A_{s, 5}^{p} X_{p} Y_{p}+A_{s, 5}^{2} Y_{p}^{2} .
\end{aligned}
$$

where the individual expressions of the coefficients can be found in $[49,25,60]$, and read:

$$
\begin{aligned}
& A_{s, 1}^{p}=\frac{\left(\omega_{s}^{2}-2 \omega_{p}^{2}\right)}{\omega_{s}^{2}\left(4 \omega_{p}^{2}-\omega_{s}^{2}\right)} g_{p p}^{s}, \\
& A_{s, 3}^{p}=\frac{-2}{\omega_{s}^{2}\left(4 \omega_{p}^{2}-\omega_{s}^{2}\right)} g_{p p}^{s}, \\
& A_{s, 5}^{p}=\frac{2}{4 \omega_{p}^{2}-\omega_{s}^{2}} g_{p p}^{s}, \\
& A_{s, 2}^{p}=A_{s, 4}^{p}=A_{s, 6}^{p}=0 .
\end{aligned}
$$

Consequently the reduced-order dynamics writes

$$
\ddot{X}_{p}+\omega_{p}^{2} X_{p}+g_{p p}^{p} X_{p}^{2}+\left(h_{p p p}^{p}+\sum_{\substack{s=1 \\ s \neq p}}^{N} g_{p s}^{p} A_{s, 1}^{p}\right) X_{p}^{3}+\left(\sum_{\substack{s=1 \\ s \neq p}}^{N} g_{p s}^{p} A_{s, 3}^{p}\right) X_{p} Y_{p}^{2}=0,
$$

and the type of nonlinearity is dictated by $\Gamma_{\mathrm{IM}}$ (where IM stands for invariant manifold):

$$
\Gamma_{\mathrm{IM}}=\frac{1}{8 \omega_{p}^{2}}\left(3 h_{p p p}^{p}-\frac{10\left(g_{p p}^{p}\right)^{2}}{3 \omega_{p}^{2}}-\sum_{\substack{s=1 \\ s \neq p}}^{N} \frac{3 \omega_{s}^{2}-8 \omega_{p}^{2}}{\omega_{s}^{2}-4 \omega_{p}^{2}} \frac{g_{p s}^{p} g_{p p}^{s}}{\omega_{s}^{2}}\right) .
$$




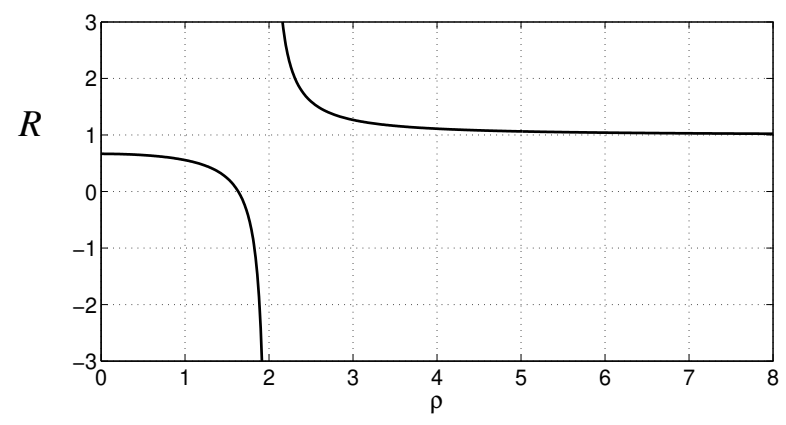

Figure 1: Correction factor $R$ defined in Eq. (26) as a function of $\rho=\omega_{s} / \omega_{p}$ the ratio between the eigenfrequencies of the slave mode $s$ and the master mode $p$. From this figure, one can conclude that the static condensation gives a correct prediction on the type of nonlinearity when $\omega_{s}>6 \omega_{p}$.

Comparing the two predictions given by Eqs. (21) and (25), one can observe that the only difference relies in the summed term, taking into account the important contributions of the slave modes to the type of nonlinearity. Interestingly, one can notice that under the slow/fast assumption, if for all $s, \omega_{s} \gg \omega_{p}$, then the asymptotic value of $\Gamma_{\mathrm{IM}}$ is equal to $\Gamma_{\mathrm{SC}}$. More precisely, assuming that a single slave mode $s$ is present in order to decompose their contributions, the ratio $R$ between the summed terms appearing in (21) and (25) simply writes

$$
R=\frac{\omega_{s}^{2}-\frac{8}{3} \omega_{p}^{2}}{\omega_{s}^{2}-4 \omega_{p}^{2}}=\frac{\rho^{2}-\frac{8}{3}}{\rho^{2}-4},
$$

where the ratio $\rho=\omega_{s} / \omega_{p}$ has been introduced. Fig. 1 shows the variations of $R$ as a function of $\rho$. First, as noted in previous articles, the formula obtained from the invariant manifold approach is correct, mainly because the reduction subspace is an NNM having the property of invariance embedded in its definition, so that trajectories simulated in the reduced subspace also exist for the complete system. Consequently Fig. 1 shows how the prediction given by static condensation diverge from the correct prediction when $R$ is different to 1 . One can observe that the static condensation does not take into account 2:1 internal resonance. In this region, when $\rho \simeq 2$, a strong coupling between the two modes exist and it becomes meaningless to define $\Gamma$ since no single-mode motions exist anymore [27]. Interestingly, Fig. 1 gives a quantitative limit from which the slow/fast assumption is fulfilled so that the static condensation can be used safely. From the figure the criterion the the eigenfrequencies reads: $\omega_{s}>6 \omega_{p}$.

\section{Slow/fast decomposition in a two dofs system}

In this section, we first begin by giving more insights to the general formulas. By restricting on a two dofs system and using asymptotic expansions to derive the first terms of the solution of the invariant manifold and the static condensation, one can realize a term-by-term comparison and contrast the similarities between the two methods.

\subsection{Asymptotic expansions}

Restricting to two degrees of freedom, the initial system simply writes:

$$
\begin{aligned}
& \ddot{X}_{1}+\omega_{1}^{2} X_{1}+f_{1}\left(X_{1}, X_{2}\right)=0, \\
& \ddot{X}_{2}+\omega_{2}^{2} X_{2}+f_{2}\left(X_{1}, X_{2}\right)=0,
\end{aligned}
$$

where $f_{p}\left(X_{1}, X_{2}\right)$ are the nonlinear internal force for $p=1,2$, which are of the polynomial type with quadratic and cubic terms so that their general expressions read:

$$
f_{p}\left(X_{1}, X_{2}\right)=g_{11}^{p} X_{1}^{2}+g_{12}^{p} X_{1} X_{2}+g_{22}^{p} X_{2}^{2}+h_{111}^{p} X_{1}^{3}+h_{112}^{p} X_{1}^{2} X_{2}+h_{122}^{p} X_{1} X_{2}^{2}+h_{222}^{p} X_{2}^{3} .
$$


Let us assume that $X_{1}$ is the master coordinate and $X_{2}$ the slave. Applying static condensation, one looks for a relationship $X_{2}=c\left(X_{1}\right)$, where $c$ is solution of Eq. (8) which can be rewritten here as:

$$
\omega_{2}^{2} c\left(X_{1}\right)+g_{11}^{2} X_{1}^{2}+g_{12}^{2} X_{1} c\left(X_{1}\right)+g_{22}^{2} c\left(X_{1}\right)^{2}+h_{111}^{2} X_{1}^{3}+h_{112}^{2} X_{1}^{2} c\left(X_{1}\right)+h_{122}^{2} X_{1} c\left(X_{1}\right)^{2}+h_{222}^{2} c\left(X_{1}\right)^{3}=0 .
$$

The solution for $c$ can be found based on an asymptotic expansion:

$$
X_{2}=c\left(X_{1}\right)=k_{2} X_{1}^{2}+k_{3} X_{1}^{3}+k_{4} X_{1}^{4}+\ldots+k_{9} X^{9}+O\left(X^{9}\right),
$$

which is stopped here at order 9 but no maximal order of the polynomial expansion can be inferred from Eq. (30) which produces new order each time the expansion for $c$ is pushed further. Term-by-term identification of the coefficients of same power gives a direct analytical solution for the $k_{i}$ coefficients introduced in (30). The quadratic and cubic coefficients read:

$$
\begin{aligned}
& k_{2}=\frac{-g_{11}^{2}}{\omega_{2}^{2}}, \\
& k_{3}=\frac{-h_{111}^{2} \omega_{2}^{2}+g_{12}^{2} g_{11}^{2}}{\omega_{2}^{4}},
\end{aligned}
$$

while the other $k_{4}$ to $k_{9}$ coefficients are given in Appendix A.

For the invariant manifolds, general expressions for the coefficients of the asymptotic expansions have already been derived in $[49,25,60]$. The two unknown functions describing the geometry of the invariant manifold can be written up to order three as:

$$
\begin{aligned}
& X_{2}=a_{2}\left(X_{1}, Y_{1}\right)=A_{11}^{1} X_{1}^{2}+A_{12}^{1} X_{1} Y_{1}+A_{22}^{1} Y_{1}^{2}+B_{111}^{1} X_{1}^{3}+B_{112}^{1} X_{1}^{2} Y_{1}+B_{122}^{1} X_{1} Y_{1}^{2}+B_{222}^{1} Y_{1}^{3}, \\
& Y_{2}=b_{2}\left(X_{1}, Y_{1}\right)=A_{11}^{2} X_{1}^{2}+A_{12}^{2} X_{1} Y_{1}+A_{22}^{2} Y_{1}^{2}+B_{111}^{2} X_{1}^{3}+B_{112}^{2} X_{1}^{2} Y_{1}+B_{122}^{2} X_{1} Y_{1}^{2}+B_{222}^{2} Y_{1}^{3} .
\end{aligned}
$$

where the general expressions for the quadratic terms can be derived from Eqs. (23), and are recalled in Appendix B, together with the full expressions for the cubic coefficients. Higher-order terms can also be found for the invariant manifold approach but their derivation leads to difficult and lengthy expressions needing for a symbolic computation processor. Comparing the expressions up to order three, one can observe that the coefficients derived from the invariant manifold approach shows singularities when internal resonances exist between the eigenfrequencies, a feature that is not expressed in the static condensation. In the next section we will show how a slow/fast assumption reveals the similarities between the two approaches.

\subsection{Slow-fast decomposition}

The general expressions given in the previous section can be simplified and directly compared if one assumes that a slow/fast decomposition is at hand between the two degrees of freedom of the system, i.e. if $\omega_{2} \gg \omega_{1}$. All expansions are also compared up to the third-order for consistency. The geometry of the stress manifold as given by the ICE method is obtained from Eq. (30) up to cubic terms and reads:

$$
X_{2}=c\left(X_{1}\right)=\frac{-g_{11}^{2}}{\omega_{2}^{2}} X_{1}^{2}+\frac{-h_{111}^{2} \omega_{2}^{2}+g_{12}^{2} g_{11}^{2}}{\omega_{2}^{4}} X_{1}^{3}
$$

The dependence on the eigenfrequencies is much more pronounced in the expressions giving the geometry of the invariant manifold in phase space, as a consequence that the inertia of the slave coordinate is not abruptly neglected. The limit values of all the coefficients $A_{i j}^{p}$ and $B_{i j k}^{p}$, for $i, j, k, p=1,2$, when $\omega_{2} \gg \omega_{1}$ are detailed in Appendix B. Consequently the nonlinear relationships between slave and master coordinates simplifies to :

$$
\begin{aligned}
& X_{2}=\frac{-g_{11}^{2}}{\omega_{2}^{2}} X_{1}^{2}+\frac{2 g_{11}^{2}}{\omega_{2}^{4}} Y_{1}^{2}+\frac{-\omega_{2}^{2} h_{111}^{2}+g_{12}^{2} g_{11}^{2}-2 g_{11}^{1} g_{11}^{2}}{\omega_{2}^{4}} X_{1}^{3}+\frac{6 \omega_{2}^{2} h_{111}^{2}-8 g_{11}^{2} g_{12}^{2}+20 g_{11}^{1} g_{11}^{2}}{\omega_{2}^{6}} X_{1} Y_{1}^{2}, \\
& Y_{2}=-\frac{2 g_{11}^{2}}{\omega_{2}^{2}} X_{1} Y_{1}+\frac{-3 \omega_{2}^{2} h_{111}^{2}+3 g_{11}^{2} g_{12}^{2}-10 g_{11}^{1} g_{11}^{2}}{\omega_{2}^{4}} X_{1}^{2} Y_{1}+\frac{6 \omega_{2}^{2} h_{111}^{2}-8 g_{11}^{2} g_{12}^{2}+20 g_{11}^{1} g_{11}^{2}}{\omega_{2}^{6}} Y_{1}^{3} .
\end{aligned}
$$


Now comparing Eqs. (33) with (34a), one can see that the quadratic terms in $X_{1}^{2}$ are the same. As announced, no dependence on the velocity master variable $Y_{1}$ exists for the stress manifold, however this dependence is proportional to $1 / \omega_{2}^{4}$ in (34a) for the quadratic term in $Y_{1}^{2}$, and can thus be considered as negligible as compared to the term in $X_{1}^{2}$, scaling as $1 / \omega_{2}^{2}$. For the cubic term in $X_{1}^{3}$, one can see that the coefficient is almost the same in the two expressions. Rewriting the difference in the last two terms in the $X_{1}^{3}$ coefficient in (34a) as $g_{11}^{2}\left(g_{12}^{2}-2 g_{11}^{1}\right)$, and recalling that the slow/fast assumption should also hold for the nonlinear stiffness so that $g_{12}^{2} \gg g_{11}^{1}$, one thus meet the conclusion that both $X_{1}^{3}$ coefficients tends to have the same values assuming the slow/fast decomposition. Finally the last cubic term in $X_{1} Y_{1}^{2}$ in (34a) is also one order of magnitude smaller, scaling as $1 / \omega_{2}^{4}$, and can thus be neglected.

Consequently, the conclusion drawn in section 2.4 holds, and the present developments show that the results given by the static condensation tend to those given by the invariant manifold approach if a slow/fast decomposition is present. The dependence on the velocity also tends to disappear, being one order of magnitude smaller. One can also see that Eq. (17), given in the general case and now specifying to

$$
b_{2} \simeq \frac{\partial a_{2}}{\partial X_{1}} Y_{1}
$$

also holds, if and only if one also assumes $g_{12}^{2} \gg g_{11}^{1}$, which is the case if the slow/fast dynamics is assumed.

Finally one can also compare the reduced-order dynamics given by the two methods, up to order three, if $\omega_{2} \gg \omega_{1}$. The dynamics of the master coordinate $X_{1}$ with the ICE method is given by Eq. (9) and reads:

$$
\ddot{X}_{1}+\omega_{1}^{2} X_{1}+g_{11}^{1} X_{1}^{2}+\left(h_{111}^{1}-\frac{g_{12}^{1} g_{11}^{2}}{\omega_{2}^{2}}\right) X_{1}^{3}=0 .
$$

On the other hand, the dynamics on the invariant manifold is given by:

$$
\ddot{X}_{1}+\omega_{1}^{2} X_{1}+g_{11}^{1} X_{1}^{2}+\left(\frac{\left(\omega_{2}^{2}-2 \omega_{1}^{2}\right)}{\omega_{2}^{2}\left(4 \omega_{1}^{2}-\omega_{2}^{2}\right)} g_{12}^{1} g_{11}^{2}+h_{111}^{1}\right) X_{1}^{3}+\left(\frac{-2}{\omega_{2}^{2}\left(4 \omega_{1}^{2}-\omega_{2}^{2}\right)} g_{12}^{1} g_{11}^{2}\right) X_{1} Y_{1}^{2}=0,
$$

which simplifies to the following with $\omega_{2} \gg \omega_{1}$ :

$$
\ddot{X}_{1}+\omega_{1}^{2} X_{1}+g_{11}^{1} X_{1}^{2}+\left(h_{111}^{1}-\frac{g_{12}^{1} g_{11}^{2}}{\omega_{2}^{2}}\right) X_{1}^{3}+\frac{2 g_{12}^{1} g_{11}^{2}}{\omega_{2}^{4}} X_{1} Y_{1}^{2}=0,
$$

Comparing Eqs. (38) with (36), one can observe that the cubic term in $X_{1}^{3}$ is exactly the same, confirming again that the stress manifold should be able to give reliable results only under the slow/fast assumption. The supplementary term in $X_{1} Y_{1}^{2}$ for the invariant manifold based ROM scales as $1 / \omega_{2}^{4}$ and should thus be negligible. The next section will illustrate all these findings on an example system.

\subsection{Example system}

In order to illustrate the previous results, the system composed of a mass connected to two nonlinear springs, is selected. Note that this system has been used in a number of studies so that numerous results are already present in the literature on this example [27, 28, 59, 61, 62].

The equation of motion for the system reads:

$$
\begin{aligned}
& \ddot{X}_{1}+\omega_{1}^{2} X_{1}+\frac{\omega_{1}^{2}}{2}\left(3 X_{1}^{2}+X_{2}^{2}\right)+\omega_{2}^{2} X_{1} X_{2}+\frac{\omega_{1}^{2}+\omega_{2}^{2}}{2} X_{1}\left(X_{1}^{2}+X_{2}^{2}\right)=0 \\
& \ddot{X}_{2}+\omega_{2}^{2} X_{2}+\frac{\omega_{2}^{2}}{2}\left(3 X_{2}^{2}+X_{1}^{2}\right)+\omega_{1}^{2} X_{1} X_{2}+\frac{\omega_{1}^{2}+\omega_{2}^{2}}{2} X_{2}\left(X_{1}^{2}+X_{2}^{2}\right)=0
\end{aligned}
$$

An advantage of the present two-dofs system is that the coefficients are fully parameterized by the two eigenfrequencies $\omega_{1}$ and $\omega_{2}$ only, since the expressions of the quadratic and cubic coupling nonlinear terms simply writes as functions of $\omega_{1}$ and $\omega_{2}$. The aim of this section is to compare the results provided 

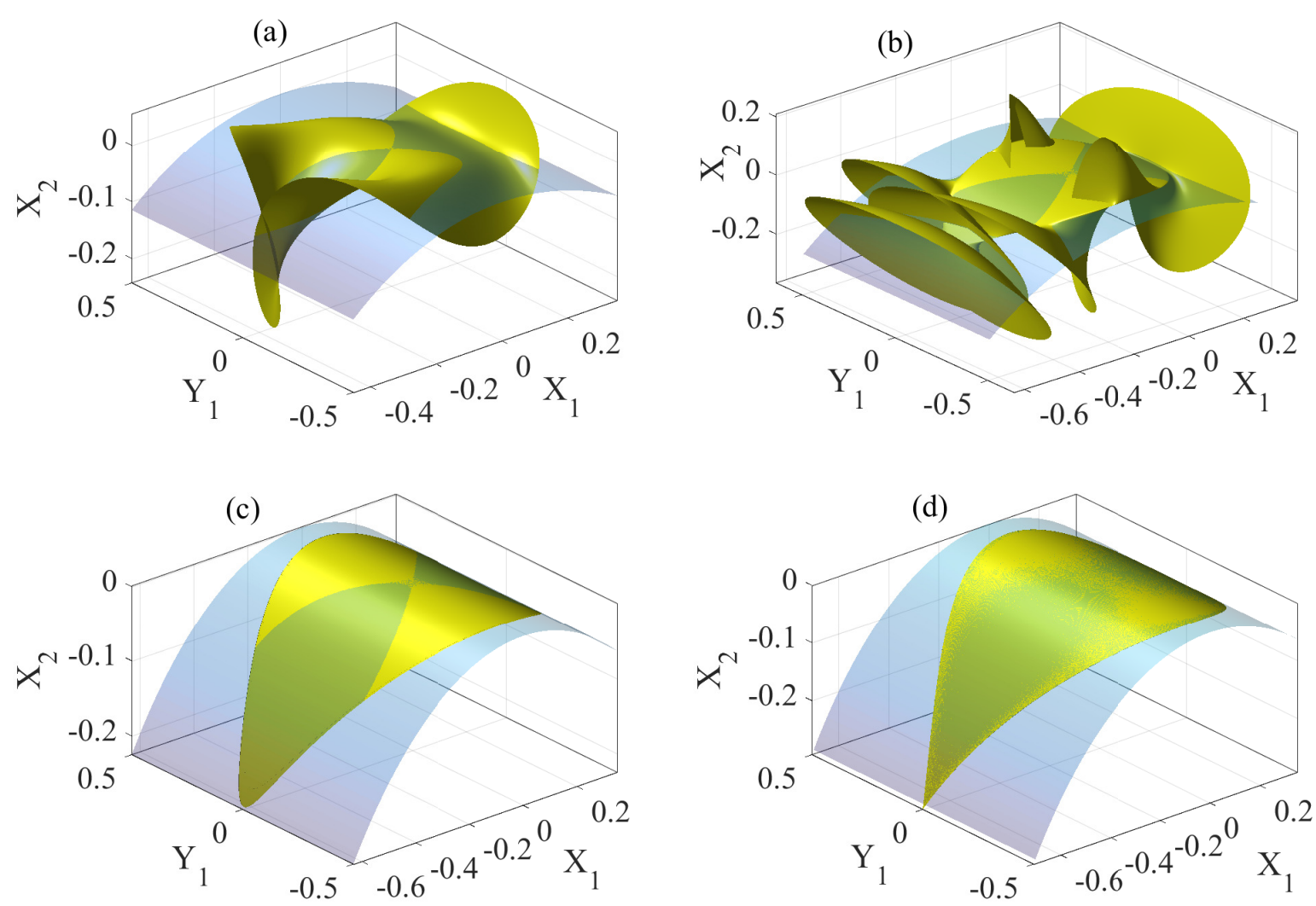

Figure 2: Comparison of stress manifold obtained with static condensation (light blue), and invariant manifold obtained from numerical continuation of periodic orbits (yellow) in phase space $\left(X_{1}, Y_{1}, X_{2}\right)$ for the two-dofs system of Eq. (39). In each figure, $\omega_{1}=1$ while $\omega_{2}$ is increased to meet the slow/fast assumption. (a) $\omega_{2}=2.5$, (b) $\omega_{2}=5$, (c) $\omega_{2}=10$ and $(\mathrm{d}): \omega_{2}=100$.

by the static condensation with those obtained with NNMs, in terms of geometry of the manifold used to reduce the dynamics, and expression of the dynamics onto this reduced subspace. As we are in a case where the model is fully known, then the ICE method is equivalent to the static condensation.

Fig. 2 shows a comparison of the stress manifold obtained from the static condensation with the invariant manifold obtained from the definition of an NNM as a collection of periodic orbits in phase space. The stress manifold has been obtained by solving numerically Eq. (8) for $s=2$, with $X_{1}$ the master coordinate. The numerical solution of this nonlinear equation allows obtaining an exact expression for the stress manifold, without any assumption on amplitudes. On the other hand, the invariant manifold is also computed numerically without any approximation so that the exact NNM is represented. The manifold is computed using numerical continuation of periodic orbits from the original system given by Eqs. (39). The continuation method uses an asymptotic-numerical method implemented in the software Manlab, where the unknowns are represented thanks to the harmonic balance method [63, 64, 65]. In order to ensure convergence, the computation has been realized with 30 harmonics.

In order to compare the results when the frequency gap between the two eigenfrequencies is increased so as to meet the slow/fast assumption, four cases are tested and reported in Fig. 2. In each case $\omega_{1}$ is set to 1 while $\omega_{2}$ is increased. As already underlined, the stress manifold does not depend on the velocity of the master variable $Y_{1}$ so that the curvatures in this direction are not well approximated. When $\omega_{2}=2.5$ and $\omega_{2}=5$, Figs. 2(a-b) clearly shows that the shape of the invariant manifold can be very complex with numerous foldings, a feature that is completely missed by the static condensation. On the other hand, from $\omega_{2}=10$, the slow/fast assumption is sufficiently met so that the geometry of the two reduced spaces tends to be equivalent. These figures allows confirming the limit exhibited in Section 2.5 on the type of nonlinearity, with a factor 6 between slave and master eigenfrequencies. Note that the shape of 
the invariant manifolds in Figs. 2(c-d) is obtained by continuation of periodic orbits and the computation has been made up to very large amplitudes where the periodic orbits saturate to the specific shape shown. This figure, obtained with the exact stress and invariant manifolds, evidences the fact that when the slow/fast assumption is verified, then the two reduction subspaces tend to share the same geometry. Note however that the geometry in phase space does not give precise information on the frequencies of the periodic orbits living inside, an issue that will be investigated later.

In order to gain more insight, further comparisons are made using the asymptotic expansions obtained in the previous section. The static condensation gives the following formula for the stress manifold up to the third order :

$$
X_{2}=c_{2}\left(X_{1}\right)=-\frac{1}{2} X_{1}^{2}+\frac{\omega_{1}^{2}}{2 \omega_{2}^{2}} X_{1}^{3} .
$$

The expansion up to order 9 can be computed using the formulas given in Appendix A which are not analytically detailed because of the lengthy expressions. In order to draw out a comparison, the geometry of the invariant manifold can be explicited by replacing in Eqs. (32) the coefficients by their values. Focusing solely on $X_{2}=a_{2}\left(X_{1}, Y_{1}\right)$ for the sake of comparison in the plane $\left(X_{1}, X_{2}\right)$, the first equation describing the geometry of the manifolds reads

$$
X_{2}=a_{2}\left(X_{1}, Y_{1}\right)=A_{11}^{1} X_{1}^{2}+A_{22}^{1} Y_{1}^{2}+B_{111}^{1} X_{1}^{3}+B_{122}^{1} X_{1} Y_{1}^{2} .
$$

The coefficients $A_{11}^{1}, A_{22}^{1}, B_{111}^{1}$ and $B_{122}^{1}$ are given below, together with their approximate value when one considers the slow/fast assumption $\omega_{2} \gg \omega_{1}$ :

$$
\begin{aligned}
& A_{11}^{1}=\frac{\omega_{2}^{2}-2 \omega_{1}^{2}}{2\left(4 \omega_{1}^{2}-\omega_{2}^{2}\right)} \stackrel{\omega_{2} \gg \omega_{1}}{\longrightarrow}-\frac{1}{2}, \\
& A_{22}^{1}=\frac{-1}{4 \omega_{1}^{2}-\omega_{2}^{2}} \stackrel{\omega_{2} \gg \omega_{1}}{\longrightarrow} \frac{1}{\omega_{2}^{2}}, \\
& B_{111}^{1}=\frac{\left(\omega_{2}^{4}-18 \omega_{1}^{4}-3 \omega_{1}^{2} \omega_{2}^{2}\right) \omega_{1}^{2} \omega_{2}^{2}}{\omega_{2}^{2}\left(4 \omega_{1}^{2}-\omega_{2}^{2}\right)\left(\omega_{2}^{2}-\omega_{1}^{2}\right)\left(\omega_{2}^{2}-9 \omega_{1}^{2}\right)} \stackrel{\omega_{2} \gg \omega_{1}}{\longrightarrow} \frac{\omega_{1}^{2}}{-\omega_{2}^{2}}, \\
& B_{122}^{1}=\frac{\left(-11 \omega_{2}^{2}-9 \omega_{1}^{2}\right) \omega_{1}^{2} \omega_{2}^{2}}{\omega_{2}^{2}\left(4 \omega_{1}^{2}-\omega_{2}^{2}\right)\left(\omega_{2}^{2}-\omega_{1}^{2}\right)\left(\omega_{2}^{2}-9 \omega_{1}^{2}\right)} \stackrel{\omega_{2} \gg \omega_{1}}{\longrightarrow} \frac{11 \omega_{1}^{2}}{\omega_{2}^{2}} .
\end{aligned}
$$

These expressions show clearly that the invariant manifold approach method gives more general results that tend to retrieve those given by static condensation at the leading order only, when a slow/fast assumption holds. Indeed, replacing the values of the coefficients $A_{11}^{1}, A_{22}^{1}, B_{111}^{1}$ and $B_{122}^{1}$ obtained with the slow/fast assumption in (41), one obtains

$$
X_{2}=a_{2}\left(X_{1}, Y_{1}\right) \simeq-\frac{1}{2} X_{1}^{2}+\frac{1}{\omega_{2}^{2}} Y_{1}^{2}-\frac{\omega_{1}^{2}}{\omega_{2}^{2}} X_{1}^{3}+\frac{11 \omega_{1}^{2}}{\omega_{2}^{2}} X_{1} Y_{1}^{2},
$$

an expression that can be directly compared to (40), showing that additional terms implying the velocities are present in the invariant manifold, but scales according to $1 / \omega_{2}^{2}$ which is assumed to be negligible. Also the cubic term in $X_{1}^{3}$ is not the same for the two methods but again is scaling according to $1 / \omega_{2}^{2}$ and is thus negligible. Consequently, the leading order term is the first quadratic term in Eqs. (40) and (43) so that a cut of both stress and invariant manifold in the plane $\left(X_{1}, X_{2}\right)$, and in the slow/fast limit, should show a parabola scaling as $-\frac{1}{2} X_{1}^{2}$.

This result is illustrated in Fig. 3. In the first panel, Fig. 3(a), the two eigenfrequencies are set as $\omega_{1}=1$ and $\omega_{2}=2.5$. The cut in the $\left(X_{1}, X_{2}\right)$ plane clearly shows that only the invariant manifold method up to order three is able to recover the correct curvature of the exact invariant manifold for moderate amplitudes of vibrations, while the static condensation gives an erroneous geometry whatever the amplitude. When increasing $\omega_{2}$ so as to meet the slow/fast assumptions, Figs. 3(b-d) shows that all curves collapses to the same parabola following the first-order term underlined before : $X_{2}=-\frac{1}{2} X_{1}^{2}$. The 

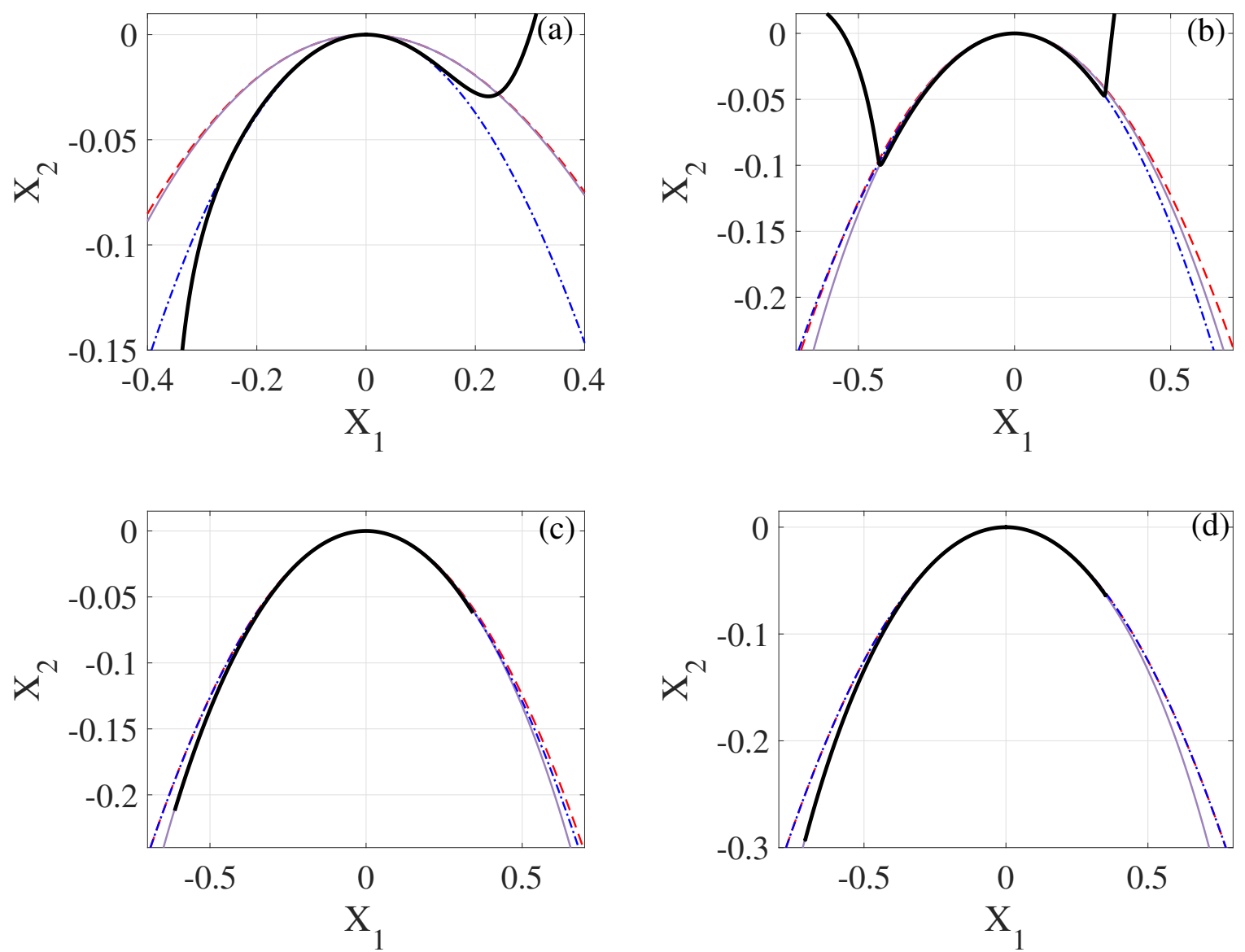

Figure 3: Comparisons of stress and invariant manifolds in the plane $\left(X_{1}, X_{2}\right)$ for the two-dofs system of Eq. (39). The static condensation up to order 3 (red dashed curve) and up to order 9 (continuous violet line) is compared to the exact invariant manifold obtained from numerical continuation of periodic orbits (black thick line, reference solution) and its third-order analytical approximation (blue dash-dotted curve). Increasing values of the frequency ratio are considered in order to meet the slow/fast assumption, with $\omega_{1}=1$, and (a): $\omega_{2}=2.5$, (b): $\omega_{2}=5$, (c) $\omega_{2}=10$ and $(\mathrm{d}): \omega_{2}=100$.

results also clearly show that static condensation up to order three is as accurate as the invariant manifold up to order three when $\omega_{2} \gg \omega_{1}$. However a better accuracy on the geometry can be obtained when pushing the development of the static condensation up to order 9, a calculation which is far easier to conduct as getting up to order 9 for the invariant manifold. We can also underline that in the range of amplitudes considered (corresponding to large amplitudes and complicated nonlinear dynamics for this system), the static condensation up to order 9 is completely equivalent to the exact value obtained by numerically solving the equation defining $c_{2}$. The exact curve for the static condensation has not been reported in Fig. 3 since it was fully merged with the ninth order approximation.

To conclude this analysis we now compare the results given by the reduced-order dynamics. Indeed, projecting the equations of motion on the correct subspace is one important point but of utmost importance is also how the periodic orbits and their frequencies are predicted by the reduced models. Using static condensation up to order three, Eq. (40), leads to the following reduced-order dynamics :

$$
\ddot{X}_{1}+\omega_{1}^{2} X_{1}+\frac{3 \omega_{1}^{2}}{2} X_{1}^{2}+\frac{\omega_{1}^{2}}{2} X_{1}^{3}=0 .
$$

This equation can be compared to the reduced dynamics given by the invariant manifold approach, 
Eq. (37), which reads, by replacing the quadratic and cubic coefficients by their values :

$$
\ddot{X}_{1}+\omega_{1}^{2} X_{1}+\frac{3 \omega_{1}^{2}}{2} X_{1}^{2}+\frac{\omega_{1}^{2}\left(4 \omega_{1}^{2}+\omega_{2}^{2}\right)}{2\left(4 \omega_{1}^{2}-\omega_{2}^{2}\right)} X_{1}^{3}+\frac{\omega_{2}^{2}}{\omega_{2}^{2}-4 \omega_{1}^{2}} X_{1} Y_{1}^{2}=0 .
$$

Using now the slow/fast assumption in Eq. (45), one obtains :

$$
\ddot{X}_{1}+\omega_{1}^{2} X_{1}+\frac{3 \omega_{1}^{2}}{2} X_{1}^{2}-\frac{\omega_{1}^{2}}{2} X_{1}^{3}+X_{1} Y_{1}^{2}=0 .
$$

Consequently even with the slow/fast assumption, one can observe in this case that the reduced-order dynamics given by the two methods, Eqs. (44) and (46) differ one from another. Again, static condensation does not give rise to any velocity-dependent terms. Since a term-by-term comparison could be misleading about the dynamics produced by the two ROMs, a better idea is to compare the outcomes of the two methods, focusing on the prediction of the type of nonlinearity, as already exemplified in section 2.5. Using the value of $\Gamma_{\mathrm{SC}}$ given in Eq. (21) and replacing the quadratic coefficients by their values, one arrives at $\Gamma_{\mathrm{SC}}=-3 / 4$ for static condensation, i.e. a constant value that do not depend on the parameter of the system. On the other hand, using Eq. (25) shows that

$$
\Gamma_{\mathrm{IM}}=\frac{-3 \omega_{1}^{2}+\omega_{2}^{2}}{4 \omega_{1}^{2}-\omega_{2}^{2}}
$$

Interestingly, the type of nonlinearity predicted by the invariant manifold approach has a divergence at the 2:1 internal resonance, a classical feature due to the strong coupling arising in the two modes in this region, and tends to -1 when $\omega_{2} \gg \omega_{1}$. This means that even if the two approaches tend to the same reduced subspaces when the slow/fast assumption holds, a persistent error in the prediction of the type of nonlinearity is given by the static condensation. This conclusion is a bit different from the one obtained in section 2.5, which is due to the particular values of the quadratic coefficients $g_{i j}^{p}$. Indeed, being fully dependent on $\omega_{1}$ and $\omega_{2}$, their relative values when applying the slow/fast assumption has a direct consequence on the results. Note also that whereas the static condensation always predicts a softening behaviour, there is a small tongue of hardening behaviour since $\Gamma_{\mathrm{IM}}$ can reach positive values. This feature thus cannot be correctly predicted by the static condensation. 

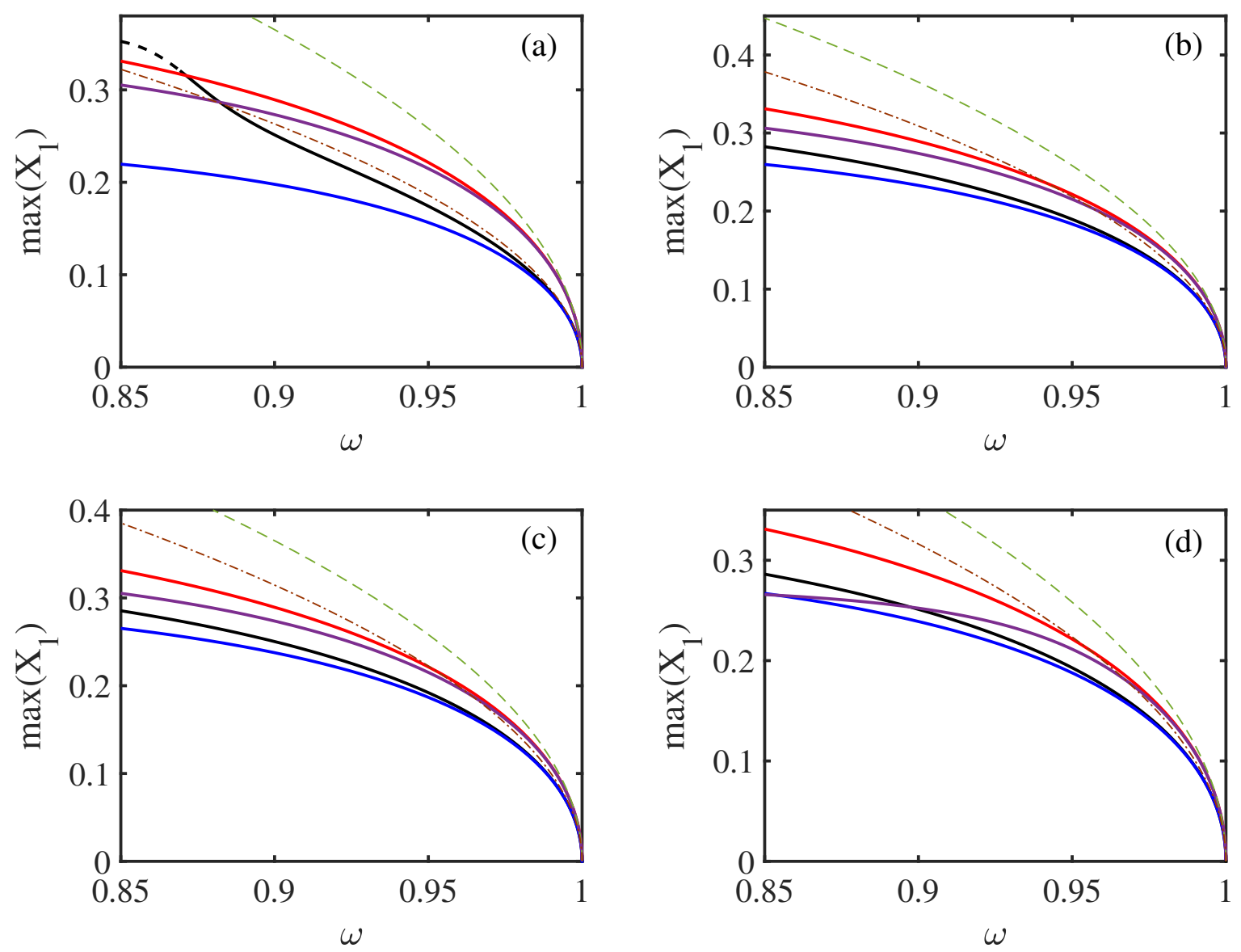

Figure 4: Comparison of backbone curves for the two-dofs system and different reduced-order models, computed with direct continuation on the equations of motion. The exact solution from the full model, Eqs. (39) (black curve with unstable part dashed), is compared to the predictions given by the static condensation up to order three, Eq. (44) (red curve) and up to order 9 (purple curve), as well as the invariant manifold approach up to order 3, Eq. (45) (blue line). Also shown as an eyeguide are the first-order backbone curves from the type of nonlinearity coefficients $\Gamma_{S C}=-3 / 4$ (dashed green line) and $\Gamma_{I M}$ given by Eq. (47) (brown dash-dotted line). The parameters are set as $\omega_{1}=1$ and increasing $\omega_{2}$ : (a): $\omega_{2}=2.5,\left(\right.$ b): $\omega_{2}=5,(\mathrm{c}): \omega_{2}=10$ and $(\mathrm{d}): \omega_{2}=100$.

These findings are illustrated in Fig. 4. One can clearly see that even with the slow/fast assumption, there is a persistent small error induced by using static condensation in order to predict the amplitudefrequency relationship, whereas the invariant manifold approach is always able to catch the first-order curvature of the backbone accurately. One can also remark that in each of the case studied, the backbone predicted using invariant manifold is close to the reference solution, except in Fig. 4(a) where the discrepancy is more proeminent and increases with amplitude. The reason for that is connected to the appearance of a more complex behaviour including a 1:2 internal resonance. Indeed, Fig. 4(a) refers to the case with $\omega_{1}=1$ and $\omega_{2}=2.5$. Since the behaviour is softening, nonlinear frequencies are decreasing and upon increasing amplitudes one tends to meet the 1:2 condition between the two nonlinear frequencies. This results in a change of behaviour of the full model solution that is not caught by the reduced order model since it is built outside such a resonance condition. Taking properly the internal resonance into account would need to have two master coordinates.

\section{Numerical examples on continuous structures}

In this section, the comparison between the implicit condensation and expansion (ICE) method and the invariant manifold is further discussed on typical beam and plate examples discretized with the finite element method, so that a better assessment of the advantages and drawbacks of each method can be 
proposed. First a beam example is selected, and reduction to a single mode is achieved with either 2D or 3D elements. Then a simply supported plate is studied, and the question of increasing the number of master modes in the ICE method is investigated. A proposition is tested in order to decrease severely the number of coefficients to be fitted when considering an important number of master modes.

\subsection{A clamped-clamped beam}

In this section, a clamped-clamped beam is investigated as a first test example. Material properties are selected as: density $\rho=7800 \mathrm{~kg} \cdot \mathrm{m}^{-3}$, Young modulus $E=2.1 e+11 \mathrm{~Pa}$, whereas the Poisson ratio is selected as $v=0$ in order to better mimic the assumptions of the theory of beams (see e.g. [41]).

To begin with, a simple case of a single master mode (the fundamental bending mode) is investigated, and the comparison between the ICE method and the NNM approach is discussed. The ICE method is derived by first applying a set of static loadings along the master mode of interest. Denoting $\phi_{1}$ the fundamental mode, a set of body forces proportional to the first mode, $\boldsymbol{F}=\beta_{1} \phi_{1}$, for increasing values of the parameter $\beta_{1}$, are imposed to the structure, i.e. to Eq. (3). One can retrieve the modal displacement by projecting the solution of the finite element procedure along $\phi_{1}$, so that a nonlinear relationship between the displacement and the scaling factors $\beta_{1}$ is numerically obtained. Fitting this relationship allows one to retrieve the nonlinear restoring force for the reduced-order model. In this paper, all the calculations have been realized with the open-source finite element software Code_aster [66]. Two different cases are selected: a thin beam meshed with 2D DKT elements, and a thick beam meshed with 3D elements, in order to underline the differences between using either surface or volume finite elements.

\subsubsection{A thin beam discretized with DKT plate elements}

We first investigate the case of a thin beam, discretized with DKT elements. The dimensions are selected as: length $L=1 \mathrm{~m}$, width $b=0.05 \mathrm{~m}$ and thickness $h=0.001 \mathrm{~m}$. The beam has been meshed with 100 elements in the length and 4 elements in the width, so as to guarantee the convergence of the first 10 eigenfrequencies of the structure. Previous investigations using the STEP method, see e.g. [46, 47, 43], show that a suitable reduced-order model can be obtained by selecting the first bending mode together with the first three even axial modes (modes number 2, 4 and 6). Indeed, the fundamental bending mode is quadratically coupled to in-plane modes and the symmetry of the problem imposes that coupling occurs only with even in-plane modes. Next, after generating this 4-mode reduced-order model with the STEP method, one can also apply either static condensation or invariant manifold approach in order to reduce the dynamics to a single dof.

The construction of the reduced model with the ICE method is illustrated in the insert in Fig. 5, where the fitting procedure for a single coordinate is shown. A total of 50 values of $\beta_{1}$ have been selected, where the load scales $\beta_{1}$ as defined from the ICE method, see section 2.2, are chosen to obtain displacements in the range of \pm 1.5 times of thickness. The curve is fitted with a polynomial expansion so that the ROM given by the ICE method reads

$$
\ddot{X}_{1}+\omega_{1}^{2} X_{1}+\gamma_{111}^{1} X_{1}^{3}=F_{1},
$$

with $\gamma_{111}^{1}=5.2310 e+09$. Note that since the beam is a flat, symmetric structure, only the cubic term appears for symmetry reasons.

Application of the STEP method using the four linear modes described above allows computing the associated quadratic and cubic coefficients. Quadratic coefficients appear due to the membrane/bending coupling [46]. Next one can apply static condensation, leading to an equation having the form of Eq. (48), with a computed coefficient now reading

$$
\tilde{\gamma}_{111}^{1}=h_{111}^{1}-\sum_{p=2}^{4} \frac{g_{1 p}^{1} g_{11}^{p}}{\omega_{p}^{2}},
$$

where the $h$ and $g$ are the modal coupling nonlinear coefficients computed from the STEP method. In this case one has $\tilde{\gamma}_{111}=5.2308 e+09$, clearly showing the equivalence between implicit and explicit condensation. Finally, starting from the ROM obtained with the STEP method, one can apply the reduction 

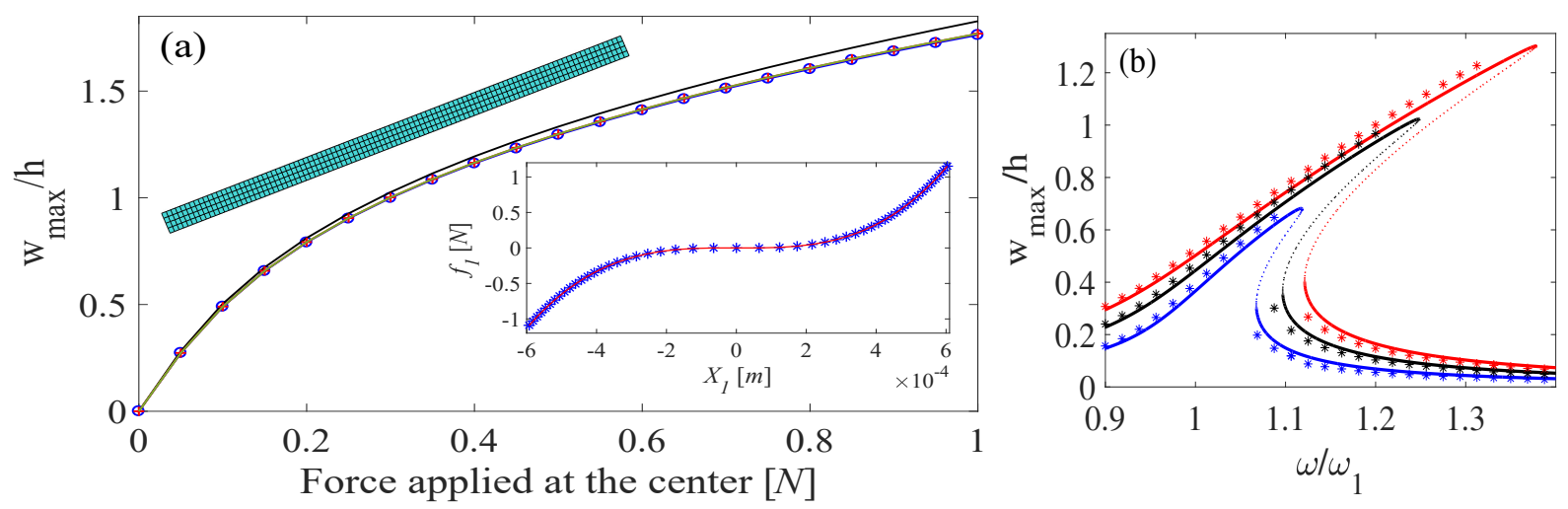

Figure 5: (a): Comparison of ROMs for a clamped-clamped beam, statically excited at the center with an increasing load. The FE solution (black line) is compared to three reduced order models: a single mode obtained with the ICE method (blue line with circle), a four mode projection using the STEP method (red line with cross), and the reduction to a single NNM from this four mode solution (green line). Insert: illustration of the fitting procedure for the ICE method: blue stars represent the outputs obtained from static applied force on the FE model, red curve is the fitted polynomial of order 3. (b): Frequency response curves of a clamped-clamped beam in the vicinity of the fundamental eigenfrequency, for three different amplitudes of the forcing: $0.00525 \mathrm{~N}$ (blue), $0.00875 \mathrm{~N}$ (black), $0.01225 \mathrm{~N}$ (red), with pointwise excitation located at center. A ROM constructed with the ICE method, Eq. (48) gives the predictions plotted with continuous lines obtained by numerical continuation, and is compared to direct time integration on the full FE model (stars).

to a single NNM, thus obtaining a reduced dynamics reading

$$
\ddot{X}_{1}+\omega_{1}^{2} X_{1}+\bar{\gamma}_{111}^{1} X_{1}^{3}+B_{111}^{1} X_{1} \dot{X}_{1}^{2}=F_{1},
$$

where $\bar{\gamma}_{111}^{1}=5.2308 e+09$ and $B_{111}^{1}=1.2506$. Comparing the values of the coefficients appearing in the reduced dynamics, on can conclude that the three methods give exactly the same results, which is in line with the findings of the previous section. Indeed, in-plane modes have very high eigenfrequencies so that the slow/fast assumption definitely holds. This is illustrated in Fig. 5 (a) with a static test, where the displacement resulting from a static force applied at the center of the beam is computed with the three reduced dynamics, and compared to the result given by the full FE solution, showing a good agreement up to 1.5 times the thickness.

A dynamical test is also performed by computing the nonlinear frequency response curve in the vicinity of the first eigenfrequency. Fig. 5 (b) shows the obtained results, where the full FE solution have been obtained using direct numerical integration (resulting in dotted points), whereas the response of the reduced models has been obtained by numerical continuation using Manlab. A Rayleigh damping of the form $C_{s}=1.34[\mathrm{M}]$ has been selected, corresponding to a damping ratio of 2 percent for the first mode.

\subsubsection{A thick beam discretized with $3 D$ elements}

The second example addresses a thick beam meshed with 3D elements, in order to illustrate some of the problems one can encounter when using 3D elements in a STEP method, and how the ICE method circumvents these issues. The convergence problems of the STEP method with 3D elements has been fully analyzed in [47]. The selected beam has the following dimensions: length $L=1 \mathrm{~m}$, width $b=$ $0.03 \mathrm{~m}$, thickness $h=0.03 \mathrm{~m}$, and is discretized with 40 elements in the length and 4 elements in the cross-section, with three-dimensional hexahedral 20 nodes finite elements. A static test with an applied force at the center of the beam is used to compare the different methods. The results are shown in Fig. 6, where the full order solution is compared with different reduced-order models, in a range of displacements up to 2.5 times the thickness. 


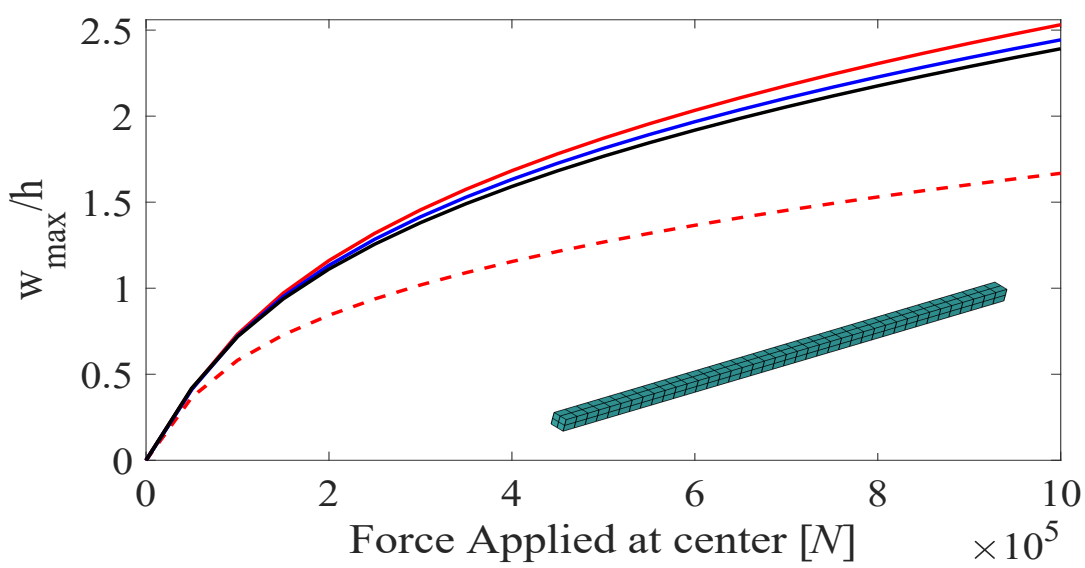

Figure 6: Static response of a thick beam discretized with 3D elements: displacement at $x=0.5 L$ versus the amplitude of the force in Newton. The reference solution, obtained with the full FE model (black line), is compared to two models using explicit static condensation: the first one composed of four eigenmodes (fundamental flexural, in-plane modes number 2,4 and 6, red dashed line), the second one composed of 87 eigenmodes (fundamental bending plus static condensation of 86 strongly coupled non-bending modes including thickness modes, red solid line); and a model obtained with the ICE method (single mode, blue solid line).

In order to illustrate the convergence problems encountered when using the linear eigenmodes as basis functions with 3D elements, a first ROM is built by using the fundamental bending mode, plus the same 3 in-plane modes identified in the previous section as the most meaningful for rendering the transverse/in-plane coupling in the case of 2D elements (axial modes 2, 4 and 6). This ROM is composed of a single oscillator equation obtained from explicit static condensation of these axial modes, where all the nonlinear coefficients are computed using the STEP method. The results plotted in red dashed line show that the ROM fails to retrieve the correct nonlinear stiffness of the beam, putting in evidence that in the case of 3D elements other major couplings arise with other non-bending modes. Indeed, as fully analyzed in [47], strong interactions occur with thickness modes having very high frequencies. Identifying these high-frequency modes is still possible in this simple case of a clamped-clamped beam with a coarse mesh, but would become impractical with more complex geometries and refined mesh. In order to achieve convergence in this case, a set of 86 non-bending modes (including in-plane and thickness modes) are needed, and the explicit static condensation of these to the first flexural mode allows retrieving the correct stiffness. On the other hand, the ICE method, which performs implicit condensation, allows finding out directly the correct result. This examples clearly stresses the main advantage of using implicit condensation, since a very rapid convergence is obtained without requiring major efforts in identifying all the coupled modes.

\subsection{A simply supported rectangular plate}

In this section we investigate a simply supported rectangular plate with the aim of developing ROMs with the ICE method including a large number of modes. Indeed, the previous section has shown the main advantage of the ICE method which, thanks to the implicit condensation, guarantees a fast convergence. However a drawback of the method consists in the multidimensional fitting procedure which is required once the set of applied static loadings have been computed. While in the case of a single master mode the fitting is easy and gives good results, when switching to a larger number of master modes two main issues arise: first, the loadings must be selected with care; second, a multivariate function has to be fitted on a multi-dimensional cloud of points. The objective of this section is to investigate these two issues on the illustrative example of the plate. In the course of the section, the dimensions of the plate are: length $L_{x}=0.254 \mathrm{~m}$, width $L_{y}=0.3556 \mathrm{~m}$, thickness $h=0.00102 \mathrm{~m}$, with material properties: density $\rho=2763 \mathrm{~kg} / \mathrm{m}^{3}$, Young modulus $E=7.3 e+10 \mathrm{~Pa}$, and Poisson ratio $v=0.3$. For the FE model, the plate was discretized with 20 elements in the length and 20 elements in the width. 


\subsubsection{Construction of multivariate ROMS based on the ICE method}

In order to build a ROM with $m$ master modes, a combination of loads associated to these is needed. The force vector to be applied in the static computation generally reads $\boldsymbol{F}= \pm \beta_{1} \phi_{1} \pm \ldots \pm \beta_{t} \phi_{t} \pm \ldots \pm$ $\beta_{m} \phi_{m}$, but numerous practical questions need to be solved for selecting the correct combinations with meaningful amplitudes. Previous studies advocated that a third-order polynomial is a correct choice in this multivariate procedure $[38,39,40,42]$. However, a number of different methods can be used for performing this STEP, as for example local interpolations. Also, deriving a functional relationship is not mandatory and a purely numerical fitting can also be used. To reduce the number of tests, we select here third-order polynomials for this fitting procedure and refer to previous studies for some details, see e.g. [42].

A key point for the computational burden is the number of static loads one has to perform before the fitting procedure. When a cubic polynomial fitting is targeted, then one can simply use, in the force vectors to be applied, combinations with only one mode, $\boldsymbol{F}= \pm \beta_{t} \phi_{t}$, two modes, $\boldsymbol{F}= \pm \beta_{t} \phi_{t} \pm \beta_{s} \phi_{s}$, or three modes can be used, since this is sufficient in order to fit quadratic and cubic polynomial terms. The number $n$ of operation associated is :

$$
n=2 m+4 \times C_{m}^{2}+8 \times C_{m}^{3},
$$

where $C_{m}^{p}=\left(\begin{array}{c}m \\ p\end{array}\right)$ is the binomial coefficient and $m$ the number of master coordinates retained in the ROM. Note that $n$ scales as $m^{3}$ and can be rapidly out of reach if ones targets ROMS with, let say, 20 to 50 modes.

Another key point is also the amplitude of the factors $\beta_{p}$ to be selected. As discussed in numerous studies, these amplitudes need not be too small so that the nonlinearity is excited, but not too large also since the FE static computation may then encounter convergence issues. Here we follow the prescription given by $[40,42,41]$. One main idea is to select the $\beta_{p}$ 's such that the resulting displacement of the structure is in a good range to excite sufficiently the nonlinearity, and the larger the value is (before having convergence issues), the better it is. A last point discussed in [42] is to add reduction factors $(1 / 2$ for combinations with two modes, and $1 / 3$ for combinations with three modes), in order to achieve a coherent range of amplitudes for each loading case. This method will be tested next and we will refer to these as the correction factors.

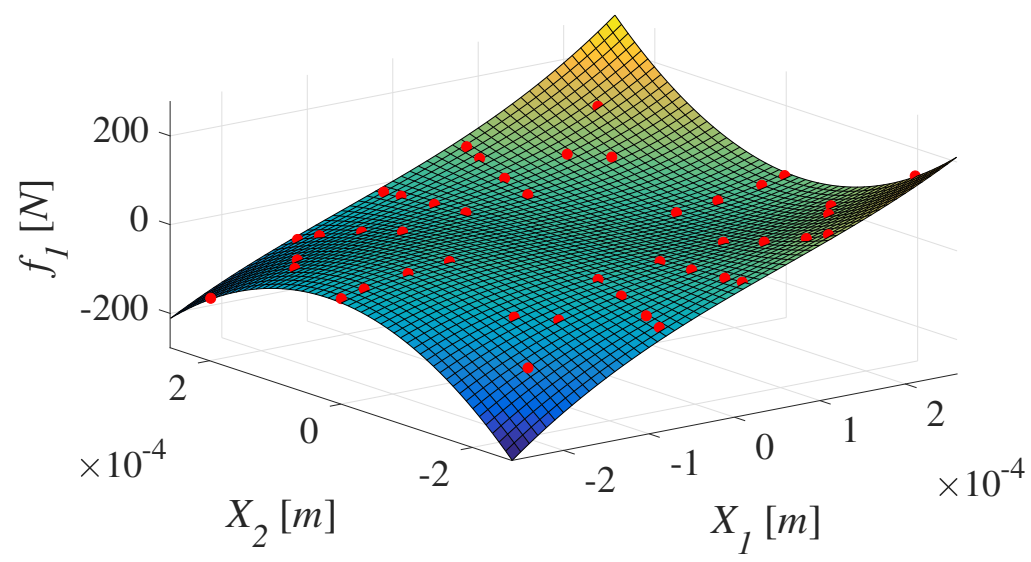

Figure 7: Illustration of the fitting procedure with two master modes, for the case of the simply supported plate. The red points represent the outputs obtained from static forces applied on the FE model with 44 load cases derived by different combination of $\pm \beta_{1} \phi_{1} \pm \beta_{4} \phi_{4}$, with $\beta$ values as $0,0.3,0.5,0.8,1$. The surface represents the fitted polynomial of order 3 .

The fitting procedure is illustrated in Fig. 7, for the case $m=2$, and for the simply supported plate studied in this section. It shows how a third-order multivariate polynomial is fitted from the points obtained from the static load cases. Augmenting the number of variables makes the problem more and 
more difficult, since the number of load cases is increasing drastically, and the fitting procedure is more and more sensitive to small variations.

When increasing the number of modes with a third-order polynomial representation of the restoring force, a specific problem is related to the number of cubic coefficients that have to be fitted (and consequently the number of applied loads needed). Indeed, the number of cubic coefficients for $m$ master modes is equal to $m \times\left(C_{m}^{3}+2 \times C_{m}^{2}+m\right)$. This number scales as $m^{4}$ and thus dramatically increases with the number of master modes. In order to reduce the computing cost, one possibility would be to identify only the monomials corresponding to the resonant terms. As known from normal form theory $[67,27,68]$, not all the monomial terms in the restoring force do have the same importance, and one can distinguish between resonant terms, that will have a strong influence on the nonlinear dynamics and bifurcations of the problems, and non-resonant monomials that could be easily cancelled thanks to a nonlinear change of coordinates. Among the resonant terms, one can also separate the trivially resonant terms, always present since linked to a trivial resonance relationship, from the resonant terms linked to an internal resonance relationship, see $[27,68]$ for more details. Trivially resonant terms are all the monomials of the form $X_{p} X_{i}^{2}$, for the $p$-th oscillator equation, with $i$ ranging from 1 to $m$. Counting only the trivially resonant terms, one can see that the number of coefficients that have to be identified scales as $m^{2}$, a drastic reduction with respect to the global estimate. This also has an implication on the number of static loads $\tilde{n}$ to apply which reduces to:

$$
\tilde{n}=2 m+4 \times C_{m}^{2},
$$

which now scales as $m^{2}$. Of course identifying only the resonant monomial terms will have drastic consequences on the fine representation of the nonlinear restoring force. However, normal form theory ensures that these monomials are the most important from the dynamical systems point of view, so that even if less precise fittings could be awaited, one is sure that no important bifurcation or nonlinear phenomena will be missed. And since the number of operations is so importantly decreased to build the ROM, it is worth testing the behaviour of such a ROM as compared to the one with all the nonlinear terms retained.

In the next sections we will discuss the choice of the scaling factors and the identification of the complete set of nonlinear terms as opposed to the reduced set of resonant ones.

\subsubsection{Simply supported rectangular plate, static excitation}

The first numerical example considers the case of the simply supported plate with a static force applied at the center. This test is more challenging than the beam case, since the number of eigenmodes needed to represent correctly the static bending of the rectangular plate is far larger. In the beam case most of the energy is concentrated in the first bending mode, while this is not the case for the plate. Indeed figure. 8 (a) shows the modal amplitudes for a static load of $70 \mathrm{~N}$ applied at the center of the plate and clearly points out that, although most of the energy is concentrated in the fundamental mode, all other ones have a meaningful contribution. As a consequence, a large number of master modes are needed to achieve convergence in the reduced-order model, making this test example interesting for testing different computational strategies.

ROMs with a maximum of 8 master modes have been tested. The number of static loads to be applied and the number of coefficients to identify are given in Table 1 for 3,5 and 8 master modes, and for the two different strategies proposed (fitting either all the monomials up to cubic order, or only the resonant terms), showing that the computational burden is already important for 8 modes, especially in the offline phase where one has to apply numerous load cases to the FE model. On the other, fitting only resonant terms leads to a drastic reduction. 


\begin{tabular}{clcc}
\hline & & Number of load set cases & Number of coefficients \\
\hline \multirow{3}{*}{ All terms } & 3 modes: & 26 & 48 \\
& 5 modes: & 130 & 175 \\
& 8 modes: & 576 & 960 \\
\hline Only & 3 modes: & 18 & 9 \\
resonant & 5 modes: & 50 & 25 \\
terms & 8 modes: & 128 & 64 \\
\hline
\end{tabular}

Table 1: The number of load cases and the number of coefficients to be fitted with the different fitting strategies proposed, with either all the monomials, or only the resonant terms.

Also, Two different strategies for selecting the $\beta_{i}$ 's coefficients in the applied load cases, have been tested, and shown in Table 2 gathering these coefficients for the first 8 modes of interest, sorted by order of increasing frequencies, and selected according to Fig. 8(a). For load case 1, the amplitudes $\beta_{i}$ have been selected such that for a linear plate, the corresponding maximal displacement for each applied force on a single mode, is equal to 2.4 times the thickness. In the simulation and due to the nonlinear restoring force, the computed static displacement is a bit smaller than the targeted one. Also in load case 1, the reduction factors ( $1 / 2$ if the forcing is a combination of two modes, and $1 / 3$ if a combination of three modes) have been applied, so that the resulting displacements from combinations of 2 and three modes are not too large. For load case 2, a different strategy has been used, without reduction factors, and with decreasing targeted linear resulting displacements, ranging from $1.5 h$ for the first mode to $0.5 h$ for mode 23.

\begin{tabular}{cccccccccc}
\hline $\begin{array}{c}\text { Scalar } \\
\text { weighting } \\
\text { factors } \beta_{i}\end{array}$ & $\beta_{1}$ & $\beta_{4}$ & $\beta_{8}$ & $\beta_{11}$ & $\beta_{12}$ & $\beta_{19}$ & $\beta_{22}$ & $\beta_{23}$ & $\begin{array}{c}\text { Reduction } \\
\text { factors }\end{array}$ \\
\hline $\begin{array}{l}\text { Load case 1 } \\
\text { Load case 2 }\end{array}$ & $5.24 \mathrm{e}-5$ & $1.95 \mathrm{e}-4$ & $3.32 \mathrm{e}-4$ & $4.73 \mathrm{e}-4$ & $4.80 \mathrm{e}-4$ & $7.56 \mathrm{e}-4$ & $8.91 \mathrm{e}-4$ & $9.07 \mathrm{e}-4$ & yes \\
\hline
\end{tabular}

Table 2: Scalar weight factors $\beta_{i}[\mathrm{~m}]$, selected for the two different load cases tested, for the simply supported plate.
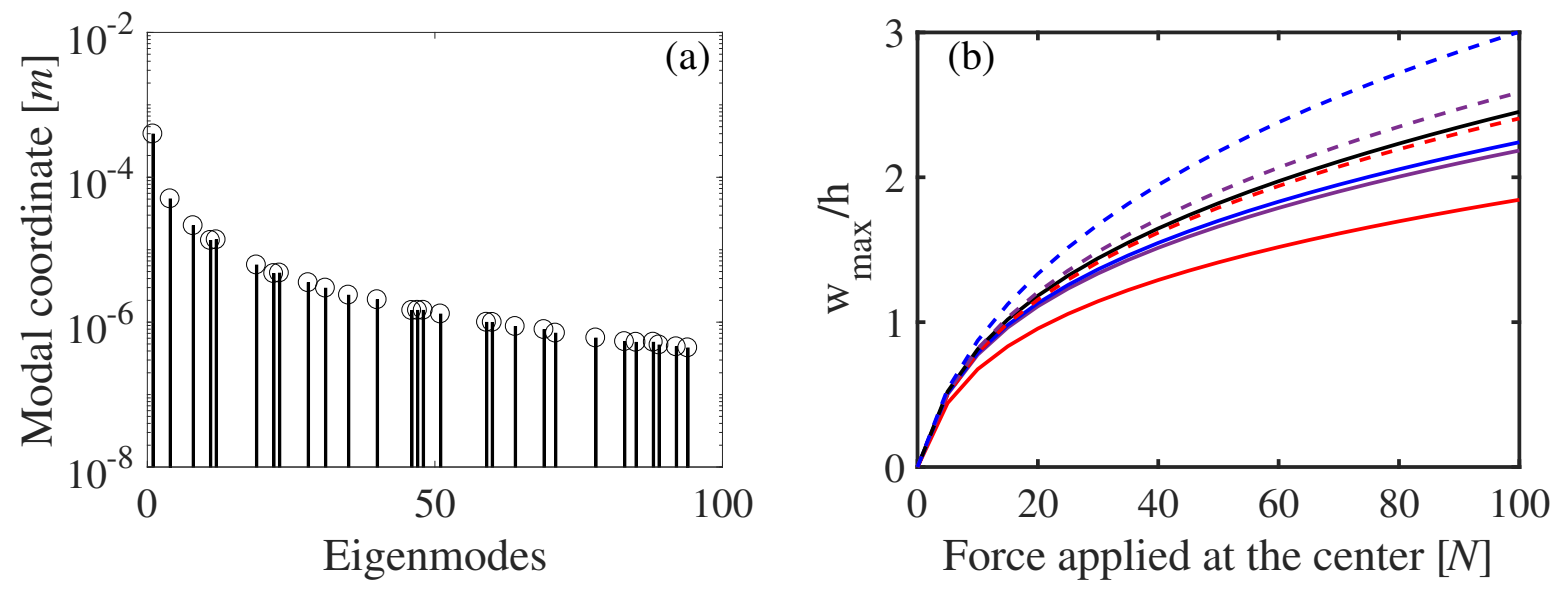

Figure 8: (a): Amplitudes of the first 100 modal coordinates $X_{p}$ as a function of the mode number, for a static load of $70 \mathrm{~N}$ applied at center of the simply supported plate. (b): Comparisons of ROMs on the static force applied at center. reference solution (full FE model, black line) is compared to the ICE method fitting with all terms (solid lines), or only with resonant terms (dashed lines). Convergence study, ICE method with all terms with 1 mode (red line), 5 modes (modes 1, 4, 8, 11 and 12, purple line) and 8 modes (adding modes 19, 22 and 23, blue line). With only resonant terms, ROM with 3 modes (1, 4 and 8, red dashed line), 5 modes (purple dashed line) and 8 modes (blue dashed line).

Fig. 8(b) shows the results obtained with ROMs generated by the ICE method with an increasing 
number of bending master modes selected in the basis. The results are given for ICE method fitted with all nonlinear terms (solid lines), or retaining only the resonant monomial terms (dotted lines). The results given by the two strategies for the load cases 1 and 2 reported in Table 2 have not been reported since they were not distinguishable, showing in this case that the effect of the selection of the $\beta_{i}$ coefficients is negligible, once the amplitude is large enough to excite the nonlinearity.

As expected, the figure highlights the slow convergence of the method. When all the nonlinear terms are fitted in the polynomial, the convergence trend is clear and the restoring force tends slowly to that of the full model. As in the case of the beam, a comparison with the STEP method has also been drawn for the plate, showing again the advantage of the implicit condensation. Indeed, in order to achieve the same accuracy as the ROM given by the ICE method with 4 master modes, it was necessary to include 141 membrane modes in the STEP method, resulting in a model with 145 modes.

The behaviour of the ROM built with only resonant terms fitted in the ICE procedure is different. First its convergence is not clearly stated with the test realized, and a larger number of master modes should be used. The conclusion is that this ROM is softer than the one with all the nonlinear terms, which appears logical. Even though most of the nonlinear terms are not meaningful in terms of the dynamical behaviour of the structure with respect to the most important phenomena such as bifurcations, retaining less terms in the polynoms leads to a softer nonlinear restoring force as that of the full model. Hence one has to keep in mind that fitting only resonant terms will produce a model that is generally not as stiff as it should be, but would be able to reproduce qualitatively the dynamic of the structure. Coming back to Fig. 8(b), it seems clear that the ROM with only resonant monomials converges to a behaviour where the internal force of the structure is not stiff enough as compared to the reference. In order to further analyze this point, the next section is devoted to dynamical simulations.

\subsubsection{Simply supported rectangular plate, dynamic response}

In this last section, we try to gain more insight on the ICE method and an increasing number of dofs focusing on the dynamic response obtained by numerical integration, for the specific case of the free vibrations of the plate when dynamically excited. The input force is applied at $\left(0.4 L_{x}, 0.35 L_{y}\right)$ and its time variation is defined by

$$
F(t)=\left\{\begin{array}{cll}
\frac{F_{\max }}{2}\left[1+\cos \left(\pi\left(t-t_{0}\right) / T_{\text {wid }}\right)\right], & \text { if } & \left|t-t_{0}\right| \leq T_{\text {wid }} \\
0, & \text { if } & \left|t-t_{0}\right|>T_{\text {wid }}
\end{array}\right.
$$

The temporal content $F(t)$ of the excitation is illustrated in Fig. 9 and depends on two parameters: $F_{\max }$ is the maximum amplitude of the strike force (in Newton), and is used to calibrate the level of geometric nonlinearities excited in the response. In the simulations, the values selected for $F_{\max }$ are $7 \mathrm{~N}, 15 \mathrm{~N}$ and $50 \mathrm{~N}$. $T_{\text {wid }}$ is the interaction time, and is used as a parameter to control the number of modes directly excited by the strike force. Indeed, denoting as $f_{m}=1 / T_{\text {wid }}$ the frequency associated to this interaction time, all the modes below $f_{m}$ are thus directly excited by the strike force. Two values of $T_{\text {wid }}$ have been selected: $T_{\text {wid }}=0.005 \mathrm{~s}$ and $T_{\text {wid }}=0.0025 \mathrm{~s}$, so as to excite the first three and eight bending eigenmodes, respectively. The first eight eigenfrequencies of the plate are listed in Table 3.

\begin{tabular}{ccccccccc}
\hline Mode & 1st & 2nd & 3rd & 4th & 5th & 6th & 7th & 8th \\
\hline Frequency[Hz] & 58.25 & 117.30 & 174.10 & 215.94 & 233.00 & 331.33 & 353.77 & 367.19 \\
\hline
\end{tabular}

Table 3: Natural frequencies of the first eight modes of the simply supported plate.

In order to test the sensitivity of the ROM constructed with ICE method to the scaling factors $\beta_{i}$ used to define the load sets, we have also selected three different strategies summarized in Table 4. Load case 1 has been selected as in the previous section, based on the advice given in [42], with a targeted linear displacement equal to 2.4 times the thickness. Note however that in the dynamic response the first eight modes (at least) are participating to the vibration so that the ROM is built for these master coordinates. Load case 2 and 3 have been selected so as to obtain the largest resulting static displacement possible in 
the full FE model without encountering convergence issues due to the appearance of large rotations. The difference lies in the use of reduction factors or not, when a combination of modes is used in the applied force. Finally, three families of ROMs are constructed, with either 3, 5 or 8 master modes, in order to test the robustness of the method with respect to an increasing number of modes.

\begin{tabular}{cccccccccc}
\hline $\begin{array}{c}\text { Scalar } \\
\text { weighting } \\
\text { factors } \beta_{t}\end{array}$ & $\beta_{1}$ & $\beta_{2}$ & $\beta_{3}$ & $\beta_{4}$ & $\beta_{5}$ & $\beta_{6}$ & $\beta_{7}$ & $\beta_{8}$ & $\begin{array}{c}\text { Reduction } \\
\text { factors }\end{array}$ \\
\hline Load case 1 & $4.34 \mathrm{e}-5$ & $8.82 \mathrm{e}-5$ & $1.31 \mathrm{e}-4$ & $1.62 \mathrm{e}-4$ & $1.75 \mathrm{e}-4$ & $2.49 \mathrm{e}-4$ & $2.67 \mathrm{e}-4$ & $2.77 \mathrm{e}-4$ & no \\
\hline Load case 2 & $3 \mathrm{e}-4$ & $3 \mathrm{e}-4$ & $3 \mathrm{e}-4$ & $3 \mathrm{e}-4$ & $3 \mathrm{e}-4$ & $3 \mathrm{e}-4$ & $3 \mathrm{e}-4$ & $3 \mathrm{e}-4$ & no \\
\hline Load case 3 & $9 \mathrm{e}-4$ & $9 \mathrm{e}-4$ & $9 \mathrm{e}-4$ & $9 \mathrm{e}-4$ & $9 \mathrm{e}-4$ & $9 \mathrm{e}-4$ & $9 \mathrm{e}-4$ & $9 \mathrm{e}-4$ & yes \\
\hline
\end{tabular}

Table 4: Scalar weighting factors $\beta_{i}$ used for computing the ROM with ICE method for the simply supported plate, for the case of the dynamical response.

The two strategies proposed in order to fit the nonlinear multivariate restoring force, i.e. by identifying only the resonant monomials or all the nonlinear terms, have been tested and compared. Note also that a few more resonant monomials have to be added to the ROM, depending on the internal resonance relationship existing between the eigenfrequencies of the structure addressed. Their selection is explained in Appendix C.

All these choices resulted in a series of 108 time simulations ( 3 values for the force amplitude, 2 values for the interaction time, 3 load cases to construct the ROM, 3 cases with different number of master modes, and two different strategies with either all nonlinear terms or only the resonant monomial terms) that have been thoroughly analyzed with different indicators, in the time and frequency domain, to understand the quality of the ROMS obtained. In all the simulation, the time step is set to be $0.0001 \mathrm{~s}$, the simulation time is $0.5 \mathrm{~s}$, the damping of the ROM is selected as $C_{s}=5+0.00001 \omega_{s}^{2}$, while in the FE model a Rayleigh damping matrix is used with $[C]=5[M]+0.00001[K]$, leading to damping ratios $\zeta$ between about 0.8 and 1.3 percent for all eight modes in the excitation band.

In the next paragraph, we show only two representative examples of the results obtained and give our comments based on the analysis of the 108 simulations. 

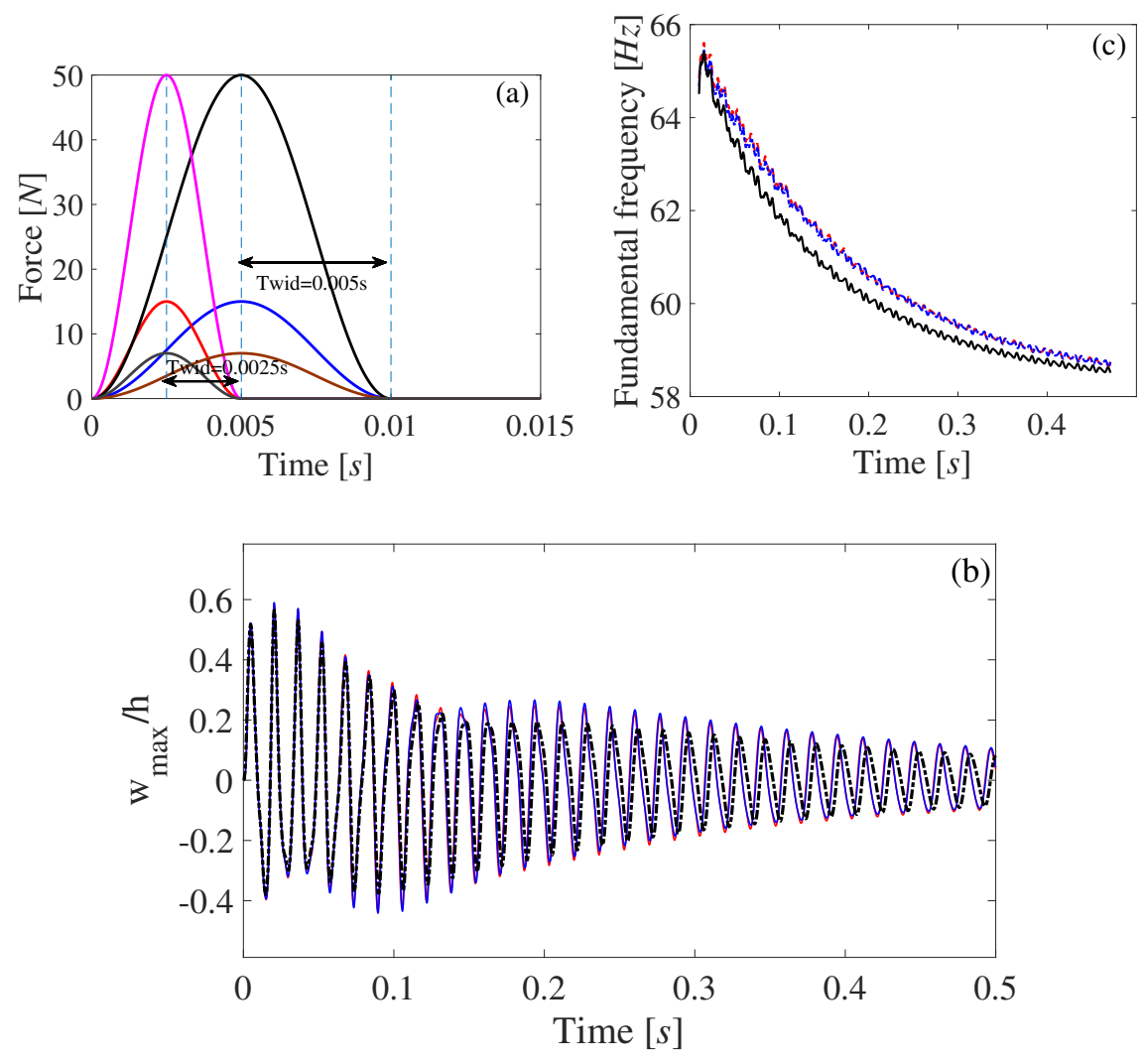

Figure 9: (a): Temporal content of the striking force given in Eq.(53), for the two different values of $T_{\text {wid }}$ and three different amplitude Fmax used in the simulations. (b): Time response of the plate subjected to a strike force. The full model (reference FE solution) is plotted in black. Displacements of two ROMS with 8 master modes and either all the nonlinear terms fitted (red curve), or only the resonant terms (blue curve). (c): Fundamental frequency variation in the case of fitting with all the terms (red) and with only resonance terms (blue); the black curve shows the reference FE solution.

The first example is a case of small excited nonlinearity $\left(F_{\max }=7 \mathrm{~N}\right)$ resulting in a maximal amplitude displacement of $0.6 h$ with $h$ the thickness of the plate. A small $T_{\text {wid }}$ is selected so that the first 8 modes are directly excited by the load, consequently two ROMs with 8 master modes are compared : one with all the polynomial terms fitted, and one with only the resonant terms. For both ROMs, load case 3 is analyzed in the following. The results are shown in Fig.9(b) for the time series of the displacement, while Fig.9(c) shows the evolution of the fundamental frequency of each displacements (full model versus the two tested ROMs), obtained directly with the yin algorithm [69]. In this case of moderate nonlinearity, it can be observed that the two ROMs are able to recover finely the temporal dynamics. The nonlinear frequency shift decreases from 66 to $58.2 \mathrm{~Hz}$ (hardening behaviour) is also very well recovered by the two ROMs. And the fact that only resonant terms are selected has no clear visible effects since the two ROMs gives hardly the same result. Regarding the different results obtained when changing the load cases reported in Table 4, it has been found that when all the terms are fitted, the three methods gives almost the same result, with a small deviation for load case 2 . When fitting only the resonant monomials, load case 3 gave the best result. The variability of the resulting ROMs with respect to load cases was more important when fitting only resonant terms, mainly because the geometric nonlinearity in the restoring force is a little less excited in this case. 
Example 2: $F_{\max }=50 \mathrm{~N}, T_{\text {wid }}=0.005 \mathrm{~s}, 3$ or 8 master modes
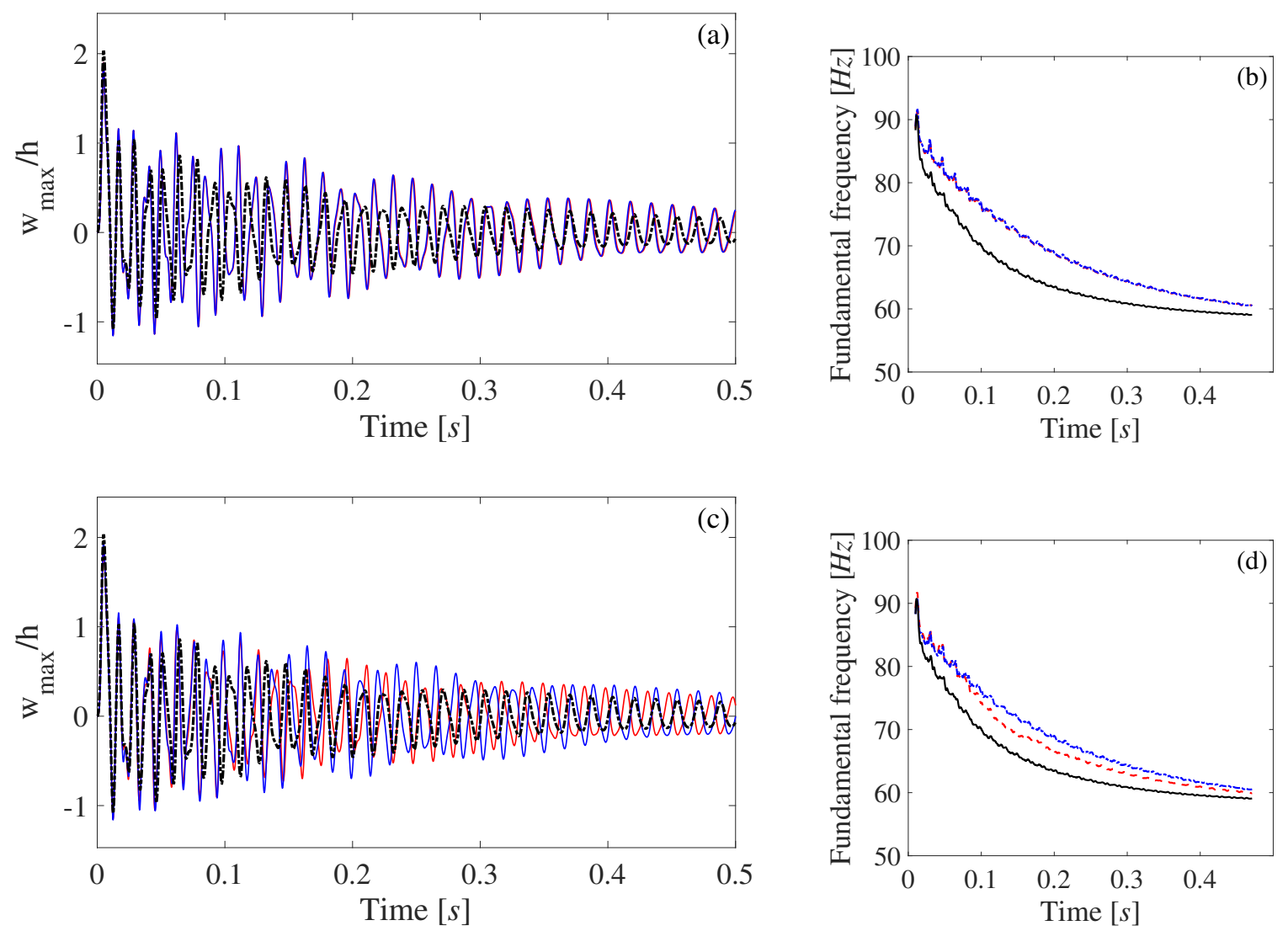

Figure 10: (a,c): Time response of the plate subjected to a strike force. (b)-(d) : variation $f$ the fundamental frequency. (a-b): 3 master modes in the ROMS, (c-d) : 8 master modes. Comparison of the full FE (reference) solution in black, ROM with all the nonlinear terms fitted (red) and only resonant terms (blue).

The second example considers a stronger nonlinearity, with $F_{\max }=50 \mathrm{~N}$, resulting in a maximum displacement of two times the thickness. A larger $T_{\text {wid }}=0.005 \mathrm{~s}$ is selected, so that only the first three modes are directly excited by the load, however since the nonlinearity is strongly excited, energy exchanges occur and other modes are then excited via nonlinear couplings. Consequently two results are reported: Fig. 10(a-b) is concerned with a ROM composed of 3 master modes, while 8 master modes are taken into account in Fig. 10(c-d). One can first observe on the time series of the displacements that whatever the ROM used, it has not been found possible to retrieve exactly the result of the full model. However the global trends are recovered in term of maximum amplitude, global decrease in time, frequency content. The decrease of the fundamental frequency is now much more pronounced with an impressive frequency shift of 50\%. Fitting with 3 modes, Fig. 10(a-b) shows that the non-resonant terms can be easily discarded to the ROM since the results are fully comparable. But none of the ROM is able to recover exactly the frequency shift.

Increasing the number of master modes to 8 shows that the two ROMs depart in the solution they compute, and the ROM with all nonlinear terms performs better, in particular with regard to the variation of the fundamental frequency. In each of these cases, the load case 3 from Table 4 has been used. It has been found that whatever the fitted model, load case 2 has the best results but very close to load case 3 used in the figure, while load case 1 gives the worst predictions.

Based on the full analysis of the 108 simulations (where also indicators on decay time, accuracy of frequency spectrum, indicators on time series and phases of the signals, have been analysed) the results show that the methods are very sensitive to either the load case, or the model used. No clear trends have been found, since some load cases could give better results for the estimation of the phase, but not on 
the decay time, just to give an example. The only clear trend found is that as long as the nonlinearity is small (example 1), and that a small number of modes (typically 3 ) are concerned, then all the methods converge and are able to finely recover the dynamical solutions. Increasing either the nonlinearity, and/or the number of modes involved in the dynamics, then all methods depart slowly from the full-order model solution. Even though all methods give generally good qualitative predictions, quantitative discrepancies appear and have a strong dependence on the input parameters and load cases. A general conclusion also is that even though fitting only the resonant terms does not give the best results when increasing the nonlinearity and the number of master modes, the results are qualitatively acceptable. Since the computational burden is so drastically reduced, the method still presents an advantage.

Our final conclusion on all these simulations is that the main drawback of the ICE method relies in this fitting procedure, which is very sensitive to a number of parameters that are difficult to control, so that it appears difficult to give clear advice on a best strategy that would work in any case and would be able to retrieve all the nonlinear characteristics of a full system.

\section{Conclusions}

This paper addressed the understanding of the implicit condensation and expansion (ICE) method, with detailed comparisons to the results provided using nonlinear normal modes (defined as invariant manifolds in phase space) so as to better understand the advantages and drawbacks of the method. One of the main advantage is to propose an implicit condensation of the non-modeled degrees of freedom (or slave variables), which has very important consequences when dealing with structures discretized with the FE approach, and for which there is no direct and simple access to the full expression of the restoring force. This main advantage leads to the fact that using the ICE method is much more efficient than using the STEP method. Indeed, the STEP method allows computing the nonlinear modal coupling coefficients, which are not dependent of the amplitude of the prescribed displacement. But the drawback is that, using the modal basis, all the known problems related to its very slow convergence for nonlinear structures, are present. This point has been clearly underlined in this contribution, in line with the results already presented by previous authors.

A second important conclusion is that the ICE method, being an implicit condensation, can not, in any case, perform better than the usual static condensation. This fact has been analysed by comparing the geometry of the reduction subspaces in phase space, together with the predictions of hardening/softening behaviour. The stress manifold, used in the ICE method to fit the nonlinear restoring force, does not depend on the velocities, and is not invariant. These two features are of great importance for producing accurate ROMS, and are embedded in the definition of NNM as invariant manifold, which produces better ROMs. In particular, our theoretical analysis shows that if a slow/fast assumption is at hand, then the results of the static condensation tend to those given by invariant manifold at the leading order only. Based on the prediction of the type of nonlinearity, a quantification of the slow/fast assumption has been proposed, with a ratio of 6 between the eigenfrequencies of master and slave coordinates.

Several numerical experiments have been addressed to better understand the behaviour of the ROMs produced with ICE method with regard to two important problems at hand when increasing the number of master modes: the strategies used to impose the prescribed forces, and the fitting procedure. In particular, it has been proposed to derive lighter ROMs by fitting only the resonant terms in the polynomial expression of the restoring force, thus drastically reducing the associated computational burden. Our main conclusion from all these experiments is that the method is generally very sensitive to the numerous parameters involved in the process (selection of $\beta_{i}$ coefficient, fitting procedure). Even though the obtained ROMs perform qualitatively well, quantitative differences are unavoidable, and are increasing with the level of nonlinearity and the number of master modes. For all these reasons, our results clearly point out that the main drawback of the method is in this fitting procedure, which needs a particular and dedicated attention. Also, all of our tests show that there is no clear guideline of which method can be used safely as giving always better results. Depending on the nonlinear characteristics one wants to reproduce accurately with a ROM (which can be for example: a correct static behaviour, a correct estimate of the nonlinear frequency shift, an accurate prediction of an important bifurcation point, a correct 
reproduction of the nonlinear couplings, etc ...), all the tested methods have shown to give, for some indicators, good results, but not for some other.

All these findings argue for using ROMs derived from the general theorems from dynamical system theory. Indeed, they are not dependent on a putative assumption of slow/fast separation between master and slave coordinates, and can be directly computed from the model, so that they belong to the class of simulation-free methods (which is not the case for e.g. POD or PGD based methods). Also, recent contributions propose their derivation in a setting that fits to the FE formulation, see $e$.g. the derivations with either the spectral submanifold method [54] or the normal form approach [55, 56]. In particular, general formula are given for an arbitrary number of master modes in [56]. As a consequence, these methods bypass the fitting procedure of the ICE method which is its main drawback, particularly when increasing the number of master modes.

\section{Acknowledgements}

The first author wish to thank China Scholarship Council (No.201806230253) for the funding of a three-year doctoral position at IMSIA, ENSTA Paris and EDF Lab. The authors also would like to thank Olivier Thomas (ENSAM Lille) for his help in the Manlab computations for obtaining the manifolds in phase space. Code_aster team and especially Ting Yu from EDF Lab are also thanked for their help in the computations.

\section{Appendix A. Analytical coefficients of asymptotic expansions for the static condensation}

In this section we give the exact analytical expressions of the coefficients obtained in the asymptotic expansion for the static condensation of a two degrees of freedom system. The functional relationship between the slave coordinate $X_{2}$ and the master one $X_{1}$ write $X_{2}=c\left(X_{1}\right)$, and $c$ is expanded in polynomial form up to order 9 following Eq. (30). Identification of like powers term give the following values for 
the coefficients $k_{2}$ to $k_{9}$ :

$k_{2}=-\left(g_{11}^{2} / \omega_{2}^{2}\right)$,

$k_{3}=\left(g_{11}^{2} g_{12}^{2}-h_{111}^{2} \omega_{2}^{2}\right) / \omega_{2}^{4}$,

$k_{4}=\left(-g_{11}^{2}\left(g_{12}^{2}\right)^{2}-\left(g_{11}^{2}\right)^{2} g_{22}^{2}+g_{12}^{2} h_{111}^{2} \omega_{2}^{2}+g_{11}^{2} h_{112}^{2} \omega_{2}^{2}\right) / \omega_{2}^{6}$,

$k_{5}=\left(g_{11}^{2}\left(g_{12}^{2}\right)^{3}+3\left(g_{11}^{2}\right)^{2} g_{12}^{2} g_{22}^{2}-\left(g_{12}^{2}\right)^{2} h_{111}^{2} \omega_{2}^{2}-2 g_{11}^{2} g_{22}^{2} h_{111}^{2} \omega_{2}^{2}-2 g_{11}^{2} g_{12}^{2} h_{112}^{2} \omega_{2}^{2}\right.$

$\left.-\left(g_{11}^{2}\right)^{2} h_{122}^{2} \omega_{2}^{2}+h_{111}^{2} h_{112}^{2} \omega_{2}^{4}\right) / \omega_{2}^{8}$,

$k_{6}=1 / \omega_{2}^{10}\left(-g_{11}^{2}\left(g_{12}^{2}\right)^{4}-6\left(g_{11}^{2}\right)^{2}\left(g_{12}^{2}\right)^{2} g_{22}^{2}-2\left(g_{11}^{2}\right)^{3}\left(g_{22}^{2}\right)^{2}+\left(g_{12}^{2}\right)^{3} h_{111}^{2} \omega_{2}^{2}\right.$

$+6 g_{11}^{2}\left(g_{12}^{2}\right) g_{22}^{2} h_{111}^{2} \omega_{2}^{2}+3 g_{11}^{2}\left(g_{12}^{2}\right)^{2} h_{112}^{2} \omega_{2}^{2}+3\left(g_{11}^{2}\right)^{2} g_{22}^{2} h_{112}^{2} \omega_{2}^{2}+$

$3\left(g_{11}^{2}\right)^{2}\left(g_{12}^{2}\right) h_{122}^{2} \omega_{2}^{2}+\left(g_{11}^{2}\right)^{3} h_{222}^{2} \omega_{2}^{2}-g_{22}^{2}\left(h_{111}^{2}\right)^{2} \omega_{2}^{4}-2\left(g_{12}^{2}\right) h_{111}^{2} h_{112}^{2} \omega_{2}^{4}-g_{11}^{2}\left(h_{112}^{2}\right)^{2} \omega_{2}^{4}-$

$\left.2 g_{11}^{2} h_{111}^{2} h_{122}^{2} \omega_{2}^{4}\right)$,

$k_{7}=1 / \omega_{2}^{12}\left(g_{11}^{2}\left(g_{12}^{2}\right)^{5}+10\left(g_{11}^{2}\right)^{2}\left(g_{12}^{2}\right)^{3} g_{22}^{2}+10\left(g_{11}^{2}\right)^{3}\left(g_{12}^{2}\right)\left(g_{22}^{2}\right)^{2}-\left(g_{12}^{2}\right)^{4} h_{111}^{2} \omega_{2}^{2}-\right.$

$12 g_{11}^{2}\left(g_{12}^{2}\right)^{2} g_{22}^{2} h_{111}^{2} \omega_{2}^{2}-6\left(g_{11}^{2}\right)^{2}\left(g_{22}^{2}\right)^{2} h_{111}^{2} \omega_{2}^{2}-4 g_{11}^{2}\left(g_{12}^{2}\right)^{3} h_{112}^{2} \omega_{2}^{2}-12\left(g_{11}^{2}\right)^{2}\left(g_{12}^{2}\right) g_{22}^{2} h_{112}^{2} \omega_{2}^{2}-$

$6\left(g_{11}^{2}\right)^{2}\left(g_{12}^{2}\right)^{2} h_{122}^{2} \omega_{2}^{2}-4\left(g_{11}^{2}\right)^{3} g_{22}^{2} h_{122}^{2} \omega_{2}^{2}-4\left(g_{11}^{2}\right)^{3}\left(g_{12}^{2}\right) h_{222}^{2} \omega_{2}^{2}+3\left(g_{12}^{2}\right) g_{22}^{2}\left(h_{111}^{2}\right)^{2} \omega_{2}^{4}+$

$3\left(g_{12}^{2}\right)^{2} h_{111}^{2} h_{112}^{2} \omega_{2}^{4}+6 g_{11}^{2} g_{22}^{2} h_{111}^{2} h_{112}^{2} \omega_{2}^{4}+3 g_{11}^{2}\left(g_{12}^{2}\right)\left(h_{112}^{2}\right)^{2} \omega_{2}^{4}+6 g_{11}^{2}\left(g_{12}^{2}\right) h_{111}^{2} h_{122}^{2} \omega_{2}^{4}+$

$\left.3\left(g_{11}^{2}\right)^{2} h_{112}^{2} h_{122}^{2} \omega_{2}^{4}+3\left(g_{11}^{2}\right)^{2} h_{111}^{2} h_{222}^{2} \omega_{2}^{4}-h_{111}^{2}\left(h_{112}^{2}\right)^{2} \omega_{2}^{6}-\left(h_{111}^{2}\right)^{2} h_{122}^{2} \omega_{2}^{6}\right)$,

$k_{8}=1 / \omega_{2}^{14}\left(-g_{11}^{2}\left(g_{12}^{2}\right)^{6}-13\left(g_{11}^{2}\right)^{2}\left(g_{12}^{2}\right)^{4} g_{22}^{2}-24\left(g_{11}^{2}\right)^{3}\left(g_{12}^{2}\right)^{2}\left(g_{22}^{2}\right)^{2}-5\left(g_{11}^{2}\right)^{4}\left(g_{22}^{2}\right)^{3}+\right.$

$2\left(g_{11}^{2}\right)^{2}\left(g_{12}^{2}\right)^{3} g_{22}^{2} \omega_{2}^{2}+6\left(g_{11}^{2}\right)^{3}\left(g_{12}^{2}\right)\left(g_{22}^{2}\right)^{2} \omega_{2}^{2}+\left(g_{12}^{2}\right)^{5} h_{111}^{2} \omega_{2}^{2}+16 g_{11}^{2}\left(g_{12}^{2}\right)^{3} g_{22}^{2} h_{111}^{2} \omega_{2}^{2}+$

$20\left(g_{11}^{2}\right)^{2}\left(g_{12}^{2}\right)\left(g_{22}^{2}\right)^{2} h_{111}^{2} \omega_{2}^{2}+5 g_{11}^{2}\left(g_{12}^{2}\right)^{4} h_{112}^{2} \omega_{2}^{2}+26\left(g_{11}^{2}\right)^{2}\left(g_{12}^{2}\right)^{2} g_{22}^{2} h_{112}^{2} \omega_{2}^{2}+10\left(g_{11}^{2}\right)^{3}\left(g_{22}^{2}\right)^{2} h_{112}^{2} \omega_{2}^{2}+$

$10\left(g_{11}^{2}\right)^{2}\left(g_{12}^{2}\right)^{3} h_{122}^{2} \omega_{2}^{2}+18\left(g_{11}^{2}\right)^{3}\left(g_{12}^{2}\right) g_{22}^{2} h_{122}^{2} \omega_{2}^{2}+18 g_{11}^{2}\left(g_{12}^{2}\right) g_{22}^{2} h_{111}^{2} h_{112}^{2} \omega_{2}^{4}-6 g_{11}^{2}\left(g_{12}^{2}\right)^{2}\left(h_{112}^{2}\right)^{2} \omega_{2}^{4}-$

$9\left(g_{11}^{2}\right)^{3}\left(g_{12}^{2}\right)^{2} h_{222}^{2} \omega_{2}^{2}+5\left(g_{11}^{2}\right)^{4} g_{22}^{2} h_{222}^{2} \omega_{2}^{2}-2 g_{11}^{2}\left(g_{12}^{2}\right)^{2} g_{22}^{2} h_{111}^{2} \omega_{2}^{4}-4\left(g_{11}^{2}\right)^{2}\left(g_{22}^{2}\right)^{2} h_{111}^{2} \omega_{2}^{4}-$

$4\left(g_{12}^{2}\right)^{2} g_{22}^{2}\left(h_{111}^{2}\right)^{2} \omega_{2}^{4}-2 g_{11}^{2}\left(g_{22}^{2}\right)^{2}\left(h_{111}^{2}\right)^{2} \omega_{2}^{4}-4\left(g_{11}^{2}\right)^{2}\left(g_{12}^{2}\right) g_{22}^{2} h_{112}^{2} \omega_{2}^{4}-4\left(g_{12}^{2}\right)^{3} h_{111}^{2} h_{112}^{2} \omega_{2}^{4}-$

$6\left(g_{11}^{2}\right)^{2} g_{22}^{2}\left(h_{112}^{2}\right)^{2} \omega_{2}^{4}-2\left(g_{11}^{2}\right)^{3} g_{22}^{2} h_{122}^{2} \omega_{2}^{4}-12 g_{11}^{2}\left(g_{12}^{2}\right)^{2} h_{111}^{2} h_{122}^{2} \omega_{2}^{4}-10\left(g_{11}^{2}\right)^{2} g_{22}^{2} h_{111}^{2} h_{122}^{2} \omega_{2}^{4}-$

$12\left(g_{11}^{2}\right)^{2}\left(g_{12}^{2}\right) h_{112}^{2} h_{122}^{2} \omega_{2}^{4}-2\left(g_{11}^{2}\right)^{3}\left(h_{122}^{2}\right)^{2} \omega_{2}^{4}-10\left(g_{11}^{2}\right)^{2}\left(g_{12}^{2}\right) h_{111}^{2} h_{222}^{2} \omega_{2}^{4}-4\left(g_{11}^{2}\right)^{3} h_{112}^{2} h_{222}^{2} \omega_{2}^{4}+$

$2 g_{11}^{2} g_{22}^{2} h_{111}^{2} h_{112}^{2} \omega_{2}^{6}+g_{22}^{2}\left(h_{111}^{2}\right)^{2} h_{112}^{2} \omega_{2}^{6}+3\left(g_{12}^{2}\right) h_{111}^{2}\left(h_{112}^{2}\right)^{2} \omega_{2}^{6}+g_{11}^{2}\left(h_{112}^{2}\right)^{3} \omega_{2}^{6}+$

$\left.3\left(g_{12}^{2}\right)\left(h_{111}^{2}\right)^{2} h_{122}^{2} \omega_{2}^{6}+6 g_{11}^{2} h_{111}^{2} h_{112}^{2} h_{122}^{2} \omega_{2}^{6}+2 g_{11}^{2}\left(h_{111}^{2}\right)^{2} h_{222}^{2} \omega_{2}^{6}\right)$,

$k_{9}=1 / \omega_{2}^{16}\left(g_{11}^{2}\left(g_{12}^{2}\right)^{7}+19\left(g_{11}^{2}\right)^{2}\left(g_{12}^{2}\right)^{5} g_{22}^{2}+64\left(g_{11}^{2}\right)^{3}\left(g_{12}^{2}\right)^{3}\left(g_{22}^{2}\right)^{2}+35\left(g_{11}^{2}\right)^{4}\left(g_{12}^{2}\right)\left(g_{22}^{2}\right)^{3}-\right.$

$2\left(g_{11}^{2}\right)^{2}\left(g_{12}^{2}\right)^{4} g_{22}^{2} \omega_{2}^{2}-6\left(g_{11}^{2}\right)^{3}\left(g_{12}^{2}\right)^{2}\left(g_{22}^{2}\right)^{2} \omega_{2}^{2}-\left(g_{12}^{2}\right)^{6} h_{111}^{2} \omega_{2}^{2}-26 g_{11}^{2}\left(g_{12}^{2}\right)^{4} g_{22}^{2} h_{111}^{2} \omega_{2}^{2}-$

$80\left(g_{11}^{2}\right)^{2}\left(g_{12}^{2}\right)^{2}\left(g_{22}^{2}\right)^{2} h_{111}^{2} \omega_{2}^{2}-20\left(g_{11}^{2}\right)^{3}\left(g_{22}^{2}\right)^{3} h_{111}^{2} \omega_{2}^{2}-6 g_{11}^{2}\left(g_{12}^{2}\right)^{5} h_{112}^{2} \omega_{2}^{2}-$

$56\left(g_{11}^{2}\right)^{2}\left(g_{12}^{2}\right)^{3} g_{22}^{2} h_{112}^{2} \omega_{2}^{2}-60\left(g_{11}^{2}\right)^{3}\left(g_{12}^{2}\right)\left(g_{22}^{2}\right)^{2} h_{112}^{2} \omega_{2}^{2}-15\left(g_{11}^{2}\right)^{2}\left(g_{12}^{2}\right)^{4} h_{122}^{2} \omega_{2}^{2}-$

$58\left(g_{11}^{2}\right)^{3}\left(g_{12}^{2}\right)^{2} g_{22}^{2} h_{122}^{2} \omega_{2}^{2}-15\left(g_{11}^{2}\right)^{4}\left(g_{22}^{2}\right)^{2} h_{122}^{2} \omega_{2}^{2}-8\left(g_{12}^{2}\right)^{3} g_{22}^{2}\left(h_{111}^{2}\right)^{2} \omega_{2}^{4}+26 g_{11}^{2}\left(g_{12}^{2}\right)\left(g_{22}^{2}\right)^{2}\left(h_{111}^{2}\right)^{2} \omega_{2}^{4}+$

$19\left(g_{11}^{2}\right)^{3}\left(g_{12}^{2}\right)^{3} h_{222}^{2} \omega_{2}^{2}-30\left(g_{11}^{2}\right)^{4}\left(g_{12}^{2}\right) g_{22}^{2} h_{222}^{2} \omega_{2}^{2}+2 g_{11}^{2}\left(g_{12}^{2}\right)^{3} g_{22}^{2} h_{111}^{2} \omega_{2}^{4}+4\left(g_{11}^{2}\right)^{2}\left(g_{12}^{2}\right)\left(g_{22}^{2}\right)^{2} h_{111}^{2} \omega_{2}^{4}+$

$4\left(g_{11}^{2}\right)^{2}\left(g_{12}^{2}\right)^{2} g_{22}^{2} h_{112}^{2} \omega_{2}^{4}+5\left(g_{12}^{2}\right)^{4} h_{111}^{2} h_{112}^{2} \omega_{2}^{4}+54 g_{11}^{2}\left(g_{12}^{2}\right)^{2} g_{22}^{2} h_{111}^{2} h_{112}^{2} \omega_{2}^{4}+$

$30\left(g_{11}^{2}\right)^{2}\left(g_{22}^{2}\right)^{2} h_{111}^{2} h_{112}^{2} \omega_{2}^{4}+10 g_{11}^{2}\left(g_{12}^{2}\right)^{3}\left(h_{112}^{2}\right)^{2} \omega_{2}^{4}+30\left(g_{11}^{2}\right)^{2}\left(g_{12}^{2}\right) g_{22}^{2}\left(h_{112}^{2}\right)^{2} \omega_{2}^{4}+$

$2\left(g_{11}^{2}\right)^{3}\left(g_{12}^{2}\right) g_{22}^{2} h_{122}^{2} \omega_{2}^{4}+20 g_{11}^{2}\left(g_{12}^{2}\right)^{3} h_{111}^{2} h_{122}^{2} \omega_{2}^{4}+58\left(g_{11}^{2}\right)^{2}\left(g_{12}^{2}\right) g_{22}^{2} h_{111}^{2} h_{122}^{2} \omega_{2}^{4}+30\left(g_{11}^{2}\right)^{2}\left(g_{12}^{2}\right)^{2} h_{112}^{2} h_{122}^{2} \omega_{2}^{4}+$

$20\left(g_{11}^{2}\right)^{3} g_{22}^{2} h_{112}^{2} h_{122}^{2} \omega_{2}^{4}+10\left(g_{11}^{2}\right)^{3}\left(g_{12}^{2}\right)\left(h_{122}^{2}\right)^{2} \omega_{2}^{4}+28\left(g_{11}^{2}\right)^{2}\left(g_{12}^{2}\right)^{2} h_{111}^{2} h_{222}^{2} \omega_{2}^{4}+20\left(g_{11}^{2}\right)^{3} g_{22}^{2} h_{111}^{2} h_{222}^{2} \omega_{2}^{4}+$

$20\left(g_{11}^{2}\right)^{3}\left(g_{12}^{2}\right) h_{112}^{2} h_{222}^{2} \omega_{2}^{4}+5\left(g_{11}^{2}\right)^{4} h_{122}^{2} h_{222}^{2} \omega_{2}^{4}-2\left(g_{22}^{2}\right)^{2}\left(h_{111}^{2}\right)^{3} \omega_{2}^{6}-2 g_{11}^{2}\left(g_{12}^{2}\right) g_{22}^{2} h_{111}^{2} h_{112}^{2} \omega_{2}^{6}-$

$10\left(g_{12}^{2}\right) g_{22}^{2}\left(h_{111}^{2}\right)^{2} h_{112}^{2} \omega_{2}^{6}-6\left(g_{12}^{2}\right)^{2} h_{111}^{2}\left(h_{112}^{2}\right)^{2} \omega_{2}^{6}-12 g_{11}^{2} g_{22}^{2} h_{111}^{2}\left(h_{112}^{2}\right)^{2} \omega_{2}^{6}-4 g_{11}^{2}\left(g_{12}^{2}\right)\left(h_{112}^{2}\right)^{3} \omega_{2}^{6}-$

$6\left(g_{12}^{2}\right)^{2}\left(h_{111}^{2}\right)^{2} h_{122}^{2} \omega_{2}^{6}-12 g_{11}^{2} g_{22}^{2}\left(h_{111}^{2}\right)^{2} h_{122}^{2} \omega_{2}^{6}-24 g_{11}^{2}\left(g_{12}^{2}\right) h_{111}^{2} h_{112}^{2} h_{122}^{2} \omega_{2}^{6}-$

$6\left(g_{11}^{2}\right)^{2}\left(h_{112}^{2}\right)^{2} h_{122}^{2} \omega_{2}^{6}-6\left(g_{11}^{2}\right)^{2} h_{111}^{2}\left(h_{122}^{2}\right)^{2} \omega_{2}^{6}-11 g_{11}^{2}\left(g_{12}^{2}\right)\left(h_{111}^{2}\right)^{2} h_{222}^{2} \omega_{2}^{6}-$

$\left.12\left(g_{11}^{2}\right)^{2} h_{111}^{2} h_{112}^{2} h_{222}^{2} \omega_{2}^{6}+h_{111}^{2}\left(h_{112}^{2}\right)^{3} \omega_{2}^{8}+3\left(h_{111}^{2}\right)^{2} h_{112}^{2} h_{122}^{2} \omega_{2}^{8}+\left(h_{111}^{2}\right)^{3} h_{222}^{2} \omega_{2}^{8}\right)$. 


\section{Appendix B. Analytical coefficients of asymptotic expansions for invariant manifold}

The full expressions of the quadratic coefficients introduced in Eq. (32) read:

$$
\begin{aligned}
& A_{11}^{1}=\frac{\left(\omega_{2}^{2}-2 \omega_{1}^{2}\right)}{\omega_{2}^{2}\left(4 \omega_{1}^{2}-\omega_{2}^{2}\right)} g_{11}^{2}, \\
& A_{22}^{1}=\frac{-2}{\omega_{2}^{2}\left(4 \omega_{1}^{2}-\omega_{2}^{2}\right)} g_{11}^{2}, \\
& A_{12}^{2}=\frac{2}{4 \omega_{1}^{2}-\omega_{2}^{2}} g_{11}^{2}, \\
& A_{12}^{1}=A_{11}^{2}=A_{22}^{2}=0,
\end{aligned}
$$

while the cubic coefficients of the invariant manifold asymptotic expansion write:

$$
\begin{aligned}
& B_{111}^{1}=\frac{\omega_{2}^{2}\left(4 \omega_{1}^{2}-\omega_{2}^{2}\right)\left(7 \omega_{1}^{2}-\omega_{2}^{2}\right) h_{111}^{2}+\left(9 \omega_{1}^{2} \omega_{2}^{2}-18 \omega_{1}^{4}-\omega_{2}^{4}\right) g_{11}^{2} g_{12}^{2}+\left(2 \omega_{2}^{4}-12 \omega_{1}^{4}-10 \omega_{1}^{2} \omega_{2}^{2}\right) g_{11}^{1} g_{11}^{2}}{\omega_{2}^{2}\left(4 \omega_{1}^{2}-\omega_{2}^{2}\right)\left(\omega_{2}^{2}-\omega_{1}^{2}\right)\left(\omega_{2}^{2}-9 \omega_{1}^{2}\right)} \\
& B_{122}^{1}=\frac{6 \omega_{2}^{2}\left(4 \omega_{1}^{2}-\omega_{2}^{2}\right) h_{111}^{2}+\left(8 \omega_{2}^{2}-18 \omega_{1}^{2}\right) g_{11}^{2} g_{12}^{2}-20 \omega_{2}^{2} g_{11}^{1} g_{11}^{2}}{\omega_{2}^{2}\left(4 \omega_{1}^{2}-\omega_{2}^{2}\right)\left(\omega_{2}^{2}-\omega_{1}^{2}\right)\left(\omega_{2}^{2}-9 \omega_{1}^{2}\right)} \\
& B_{112}^{2}=\frac{3 \omega_{2}^{2}\left(4 \omega_{1}^{2}-\omega_{2}^{2}\right)\left(3 \omega_{1}^{2}-\omega_{2}^{2}\right) h_{111}^{2}+\left(11 \omega_{1}^{2} \omega_{2}^{2}-3 \omega_{2}^{4}-18 \omega_{1}^{4}\right) g_{11}^{2} g_{12}^{2}-10 \omega_{2}^{2}\left(3 \omega_{1}^{2}-\omega_{2}^{2}\right) g_{11}^{1} g_{11}^{2}}{\omega_{2}^{2}\left(4 \omega_{1}^{2}-\omega_{2}^{2}\right)\left(\omega_{2}^{2}-\omega_{1}^{2}\right)\left(\omega_{2}^{2}-9 \omega_{1}^{2}\right)} \\
& B_{222}^{2}=B_{122}^{1}, \\
& B_{111}^{2}=B_{122}^{2}=B_{112}^{1}=B_{222}^{1}=0 .
\end{aligned}
$$

Assuming a slow/fast decomposition, i.e. $\omega_{2} \gg \omega_{1}$ for this two-dofs system, we give here the limited values of all the coefficients $A_{i j}^{p}$ and $B_{i j k}^{p}(i, j, k, p=1,2)$ appearing in Eqs. (32). We begin with the quadratic coefficients $A_{i j}^{p}$ :

$$
\begin{aligned}
& A_{11}^{1} \stackrel{\omega_{2} \gg \omega_{1}}{\longrightarrow}-\frac{g_{11}^{2}}{\omega_{2}^{2}}, \\
& A_{22}^{1} \stackrel{\omega_{2} \gg \omega_{1}}{\longrightarrow} \frac{2 g_{11}^{2}}{\omega_{2}^{4}}, \\
& A_{12}^{1} \stackrel{\omega_{2} \gg \omega_{1}}{\longrightarrow}-\frac{2 g_{11}^{2}}{\omega_{2}^{2}}, \\
& A_{12}^{1}=A_{11}^{2}=A_{22}^{2}=0 .
\end{aligned}
$$

For the cubic coefficients we have :

$$
\begin{aligned}
& B_{111}^{1} \stackrel{\omega_{2} \gg \omega_{1}}{\longrightarrow} \frac{-\omega_{2}^{2} h_{111}^{2}+g_{11}^{2} g_{12}^{2}-2 g_{11}^{1} g_{11}^{2}}{\omega_{2}^{4}}, \\
& B_{122}^{1} \stackrel{\omega_{2} \gg \omega_{1}}{\longrightarrow} \frac{6 \omega_{2}^{2} h_{111}^{2}-8 g_{11}^{2} g_{12}^{2}+20 g_{11}^{1} g_{11}^{2}}{\omega_{2}^{6}}, \\
& B_{112}^{2} \stackrel{\omega_{2} \gg \omega_{1}}{\longrightarrow} \frac{-3 \omega_{2}^{2} h_{111}^{2}+3 g_{11}^{2} g_{12}^{2}-10 g_{11}^{1} g_{11}^{2}}{\omega_{2}^{4}}, \\
& B_{222}^{2}=B_{122}^{1}, \\
& B_{111}^{2}=B_{122}^{2}=B_{112}^{1}=B_{222}^{1}=0 .
\end{aligned}
$$




\section{Appendix C. Identification of resonant monomials for the simply supported plate}

Apart from the trivially resonant terms that always need to be taken into account in the reduced model, whatever the relationships between the eigenfrequencies, some additional monomials shall also be taken into account if the eigenfrequencies of the studied structure present internal resonance relationships. Note that since a flat symmetric structure is studied, only the third-order internal resonance relationships need to be verified, since the restoring force shall not contain quadratic terms. For the selected rectangular plate with simply supported boundary conditions, whose first eight eigenfrequencies are given in Table 4, one can observe that the following third-order relationships of closeness to internal resonance are verified:

$$
\begin{aligned}
& \omega_{3} \approx 3 \omega_{1} \\
& \omega_{3} \approx 2 \omega_{2}-\omega_{1} \\
& \omega_{5} \approx 2 \omega_{1}+\omega_{2} \\
& \omega_{5} \approx 2 \omega_{3}-\omega_{2} \\
& \omega_{5} \approx \omega_{2}+\omega_{3}-\omega_{1}, \\
& \omega_{6} \approx 2 \omega_{1}+\omega_{4} \\
& \omega_{6} \approx \omega_{3}+\omega_{4}-\omega_{1} \\
& \omega_{6} \approx \omega_{4}+\omega_{5}-\omega_{2} \\
& \omega_{7} \approx 3 \omega_{2}
\end{aligned}
$$

This means in particular that all the resonant monomial terms corresponding to these relationships need to be added to the ROM. For example from $\omega_{3} \approx 3 \omega_{1}$, one has to take into account a term of the form $h_{111}^{3} X_{1}^{3}$ in the equation for $X_{3}$, and a term $h_{113}^{1} X_{1}^{2} X_{3}$ in the equation for $X_{1}$. If the relationships involves three eigenfrequencies like $\omega_{3} \approx 2 \omega_{2}-\omega_{1}$, then three additional terms are needed: $h_{122}^{3} X_{1} X_{2}^{2}$ in the equation for $X_{3}, h_{223}^{1} X_{2}^{2} X_{3}$ in the equation for $X_{1}$, and $h_{123}^{2} X_{1} X_{2} X_{3}$ in the equation for $X_{2}$. Finally four terms are needed if the internal resonance relationship involves 4 eigenfrequencies. Consequently, in the ROM composed of 8 master mode, a number of 28 more monomials have been added.

\section{References}

[1] Y. C. Fung and P. Tong. Classical and Computational Solid Mechanics. World Scientific, River Edge, New Jersey, 2001.

[2] W. Soedel. Vibrations of Shells and Plates. Marcel Dekker, inc., New York, 1981.

[3] A. H. Nayfeh and P. F. Pai. Linear and nonlinear structural mechanics. Wiley, New-York, 2004.

[4] A. H. Nayfeh and D. T. Mook. Nonlinear oscillations. John Wiley \& sons, New-York, 1979.

[5] A. H. Nayfeh. Nonlinear interactions: analytical, computational and experimental methods. Wiley series in nonlinear science, New-York, 2000.

[6] P. B. Gonçalves. Axisymmetric vibrations of imperfect shallow spherical caps under pressure loading. Journal of Sound and Vibration, 174(2):249-260, 1994.

[7] M. Amabili. Nonlinear vibrations and stability of shells and plates. Cambridge University Press, 2008.

[8] A. Abe, Y. Kobayashi, and G. Yamada. Nonlinear dynamic behaviors of clamped laminated shallow shells with one-to-one internal resonance. Journal of Sound and Vibration, 304(3-5):957-968, 2007. 
[9] M. Amabili, F. Pellicano, and A. F. Vakakis. Nonlinear vibrations and multiple resonances of fluidfilled, circular shells, part I: equations of motion and numerical results. ASME Journal of Vibration and Acoustics, 122:346-354, 2000.

[10] O. Thomas, C. Touzé, and A. Chaigne. Non-linear vibrations of free-edge thin spherical shells: modal interaction rules and 1:1:2 internal resonance. International Journal of Solids and Structures, 42(11-12):3339-3373, 2005.

[11] J. Awrejcewicz, V. A. Krysko, and T. V. Shchekaturova. Transition from regular to chaotic vibrations of spherical and conical axially-symmetric shells. International Journal of Structural Stability and Dynamics, 5(3):359-385, 2005.

[12] C. Touzé, O. Thomas, and M. Amabili. Transition to chaotic vibrations for harmonically forced perfect and imperfect circular plates. International Journal of Non-linear Mechanics, 46(1):234$246,2011$.

[13] A. Boudaoud, O. Cadot, B. Odille, and C. Touzé. Observation of wave turbulence in vibrating plates. Physical Review Letters, 100:234504, 2008.

[14] C. Touzé, S. Bilbao, and O. Cadot. Transition scenario to turbulence in thin vibrating plates. Journal of Sound and Vibration, 331(2):412-433, 2012.

[15] O. Cadot, M. Ducceschi, T. Humbert, B. Miquel, N. Mordant, C. Josserand, and C. Touzé. Wave turbulence in vibrating plates. Chapman and Hall/CRC, 2016. in C. Skiadas (editor) : Handbook of Applications of Chaos theory.

[16] R. K. Kapania and C. Byun. Reduction methods based on eigenvectors and Ritz vectors for nonlinear transient analysis. Computational Mechanics, 11(1):65-82, 1993.

[17] P. Krysl, S. Lall, and J.E. Marsden. Dimensional model reduction in non-linear finite element dynamics of solids and structures. International Journal for numerical methods in engineering, 51:479-504, 2001.

[18] M. Amabili, A. Sarkar, and M. P. Païdoussis. Reduced-order models for nonlinear vibrations of cylindrical shells via the proper orthogonal decomposition method. Journal of Fluids and Structures, 18(2):227-250, 2003.

[19] G. Kerschen, J.C. Golinval, A.F. Vakakis, and L.A. Bergman. The method of proper orthogonal decomposition for dynamical characterization and order reduction of mechanical systems: an overview. Nonlinear Dynamics, 41:147-169, 2005.

[20] F. Chinesta, P. Ladevèze, and E. Cueto. A short review on model order reduction based on proper generalized decomposition. Archives of Computational Methods in Engineering, 18(4):395, 2011.

[21] L. Meyrand, E. Sarrouy, B. Cochelin, and G. Ricciardi. Nonlinear normal mode continuation through a proper generalized decomposition approach with modal enrichment. Journal of Sound and Vibration, 443:444 - 459, 2019.

[22] O. Thomas and S. Bilbao. Geometrically nonlinear flexural vibrations of plates: In-plane boundary conditions and some symmetry properties. Journal of Sound and Vibration, 315(3):569-590, 2008.

[23] M. Amabili. A comparison of shell theories for large-amplitude vibrations of circular cylindrical shells: Lagrangian approach. Journal of Sound and Vibration, 264:1091-1125, 2003.

[24] S. W. Shaw and C. Pierre. Non-linear normal modes and invariant manifolds. Journal of Sound and Vibration, 150(1):170-173, 1991. 
[25] N. Boivin, C. Pierre, and S. Shaw. Non-linear normal modes, invariance, and modal dynamics approximations of non-linear systems. Nonlinear Dynamics, 8:315-346, 1995.

[26] E. Pesheck, C. Pierre, and S. Shaw. A new Galerkin-based approach for accurate non-linear normal modes through invariant manifolds. Journal of Sound and Vibration, 249(5):971-993, 2002.

[27] C. Touzé, O. Thomas, and A. Chaigne. Hardening/softening behaviour in non-linear oscillations of structural systems using non-linear normal modes. Journal of Sound and Vibration, 273(1-2):77$101,2004$.

[28] C. Touzé and M. Amabili. Non-linear normal modes for damped geometrically non-linear systems: application to reduced-order modeling of harmonically forced structures. Journal of Sound and Vibration, 298(4-5):958-981, 2006.

[29] M. Amabili and C. Touzé. Reduced-order models for non-linear vibrations of fluid-filled circular cylindrical shells: comparison of pod and asymptotic non-linear normal modes methods. Journal of Fluids and Structures, 23(6):885-903, 2007.

[30] G. Haller and S. Ponsioen. Nonlinear normal modes and spectral submanifolds: existence, uniqueness and use in model reduction. Nonlinear Dynamics, 86(3):1493-1534, 2016.

[31] S. Ponsioen, T. Pedergnana, and G. Haller. Automated computation of autonomous spectral submanifolds for nonlinear modal analysis. Journal of Sound and Vibration, 420:269 - 295, 2018.

[32] M. P. Mignolet, A. Przekop, S. A. Rizzi, and S. M. Spottswood. A review of indirect/nonintrusive reduced order modeling of nonlinear geometric structures. Journal of Sound and Vibration, 332:2437-2460, 2013.

[33] A. Lazarus, O. Thomas, and J.-F. De. Finite element reduced order models for nonlinear vibrations of piezoelectric layered beams with applications to nems. Finite Elements in Analysis and Design, 49(1):35-51, 2012.

[34] C. Touzé, M. Vidrascu, and D. Chapelle. Direct finite element computation of non-linear modal coupling coefficients for reduced-order shell models. Computational Mechanics, 54(2):567-580, 2014.

[35] A.A. Muravyov and S.A. Rizzi. Determination of nonlinear stiffness with application to random vibration of geometrically nonlinear structures. Computers and Structures, 81:1513-1523, 2003.

[36] M. Mignolet and C. Soize. Stochastic reduced-order models for uncertain geometrically nonlinear dynamical systems. Computer Methods in AppL. Mech. Engrg., 197:3951-3963, 2008.

[37] R. Perez, X. Q. Wang, and M. P. Mignolet. Nonintrusive Structural Dynamic Reduced Order Modeling for Large Deformations: Enhancements for Complex Structures. Journal of Computational and Nonlinear Dynamics, 9(3), 2014.

[38] M. Mc Ewan, J. Wright, J. Cooper, and A. Leung. A finite element/modal technique for nonlinear plate and stiffened panel response prediction. In 19th AIAA Applied Aerodynamics Conference, 2001.

[39] M. I. Mc Ewan. A combined modal/finite element technique for the non-linear dynamic simulation of aerospace structures. PhD thesis, University of Manchester, 2001.

[40] J. J. Hollkamp and R. W. Gordon. Reduced-order models for non-linear response prediction: Implicit condensation and expansion. Journal of Sound and Vibration, 318:1139-1153, 2008.

[41] A. Frangi and G. Gobat. Reduced order modelling of the non-linear stiffness in mems resonators. International Journal of Non-Linear Mechanics, 116:211 - 218, 2019. 
[42] R. J. Kuether, B. J. Deaner, J. J. Hollkamp, and M. S. Allen. Evaluation of geometrically nonlinear reduced-order models with nonlinear normal modes. AIAA Journal, 53(11):3273-3285, 2015.

[43] C. S. M. Sombroek, P. Tiso, L. Renson, and G. Kerschen. Numerical computation of nonlinear normal modes in a modal derivative subspace. Computers \& Structures, 195:34 - 46, 2018.

[44] A. Vizzaccaro, L. Salles, and C. Touzé. Comparison of nonlinear mappings for reduced-order modelling of vibrating structures: normal form theory and quadratic manifold method with modal derivatives. Nonlinear Dynamics, accepted for publication, 2020.

[45] L. E. Malvern. Introduction to the mechanics of a continuous medium. Pearson, New-York, 1977.

[46] A. Givois, A. Grolet, O. Thomas, and J.-F. Deü. On the frequency response computation of geometrically nonlinear flat structures using reduced-order finite element models. Nonlinear Dynamics, 97(2):1747-1781, 2019.

[47] A. Vizzaccaro, A. Givois, P. Longobardi, Y. Shen, J.-F. Deü, L. Salles, C. Touzé, and O. Thomas. Non-intrusive reduced order modelling for the dynamics of geometrically nonlinear flat structures using three-dimensional finite elements. Computational Mechanics, accepted for publication, 2020.

[48] J. J. Hollkamp, R. W. Gordon, and S. M. Spottswood. Non-linear modal models for sonic fatigue response prediction: a comparison of methods. Journal of Sound and Vibration, 284:1145-1163, 2005.

[49] S. W. Shaw and C. Pierre. Normal modes for non-linear vibratory systems. Journal of Sound and Vibration, 164(1):85-124, 1993.

[50] S. W. Shaw and C. Pierre. Normal modes of vibration for non-linear continuous systems. Journal of Sound and Vibration, 169(3):85-124, 1994.

[51] A. Kelley. The stable, center-stable, center, center-unstable and unstable manifolds. Journal of Differential Equations, 3:546-570, 1967.

[52] J. Guckenheimer and P. Holmes. Nonlinear oscillations, dynamical systems and bifurcations of vector fields. Springer-Verlag, New-York, 1983.

[53] P. Apiwattanalunggarn, C. Pierre, and D. Jiang. Finite-element-based nonlinear modal reduction of a rotating beam with large-amplitude motion. Journal of Vibration and Control, 9:235-263, 2003.

[54] Z. Veraszto, S. Ponsioen, and G. Haller. Explicit third-order model reduction formulas for general nonlinear mechanical systems. Journal of Sound and Vibration, 468:115039, 2020.

[55] A. Vizzaccaro, Y. Shen, L. Salles, and C. Touzé. Model order reduction methods based on normal form for geometrically nonlinear structures: a direct approach. In proc. of Euromech Non-linear Dynamics Conference, ENOC 2020, Lyon, July 2020.

[56] A. Vizzaccaro Y. Shen, L. Salles, J. Blahos, and C. Touzé. Direct computation of normal form for reduced-order models of finite element nonlinear structures. CMAME, submitted for publication, 2020.

[57] D. Jiang, C. Pierre, and S. Shaw. The construction of non-linear normal modes for systems with internal resonance. International Journal of Non-linear Mechanics, 40(5):729-746, 2005.

[58] F. Blanc, C. Touzé, J.-F. Mercier, K. Ege, and A.-S. Bonnet Ben-Dhia. On the numerical computation of nonlinear normal modes for reduced-order modelling of conservative vibratory systems. Mechanical Systems and Signal Processing, 36(2):520 - 539, 2013. 
[59] L. Renson, G. Kerschen, and B. Cochelin. Numerical computation of nonlinear normal modes in mechanical engineering. Journal of Sound and Vibration, 364:177 - 206, 2016.

[60] E. Pesheck, N. Boivin, C. Pierre, and S. W. Shaw. Nonlinear modal analysis of structural systems using multi-mode invariant manifolds. Nonlinear Dynamics, 25(1):183-205, 2001.

[61] Thomas Breunung and George Haller. Explicit backbone curves from spectral submanifolds of forced-damped nonlinear mechanical systems. Proceedings of the Royal Society A: Mathematical, Physical and Engineering Sciences, 474(2213):20180083, 2018.

[62] X. Liu and D. J. Wagg. Simultaneous normal form transformation and model-order reduction for systems of coupled nonlinear oscillators. Proceedings of the Royal Society A: Mathematical, Physical and Engineering Sciences, 475(2228):20190042, 2019.

[63] B. Cochelin and C. Vergez. A high order purely frequency-based harmonic balance formulation for continuation of periodic solutions. Journal of Sound and Vibration, 324(1):243 - 262, 2009.

[64] A. Lazarus and O. Thomas. A harmonic-based method for computing the stability of periodic solutions of dynamical systems. Comptes Rendus Mécanique, 338(9):510 - 517, 2010.

[65] L. Guillot, B. Cochelin, and C. Vergez. A generic and efficient taylor seriesbased continuation method using a quadratic recast of smooth nonlinear systems. International Journal for Numerical Methods in Engineering, 119(4):261-280, 2019.

[66] Électricité de France. code_aster, accessed February 10, 2020. https://www.code-aster.org/.

[67] G. Iooss and M. Adelmeyer. Topics in bifurcation theory. World scientific, New-York, 1998. second edition.

[68] C. Touzé. Normal form theory and nonlinear normal modes: theoretical settings and applications. In G. Kerschen, editor, Modal Analysis of nonlinear Mechanical Systems, pages 75-160, New York, NY, 2014. Springer Series CISM courses and lectures, vol. 555.

[69] A. de Cheveigné and H. Kawahara. Yin, a fundamental frequency estimator for speech and music. The Journal of the Acoustical Society of America, 111(4):1917-1930, 2002. 